\title{
Benchmark
}

Les réseaux sociaux

en bibliothèque de lecture publique

\section{Etude comparative}

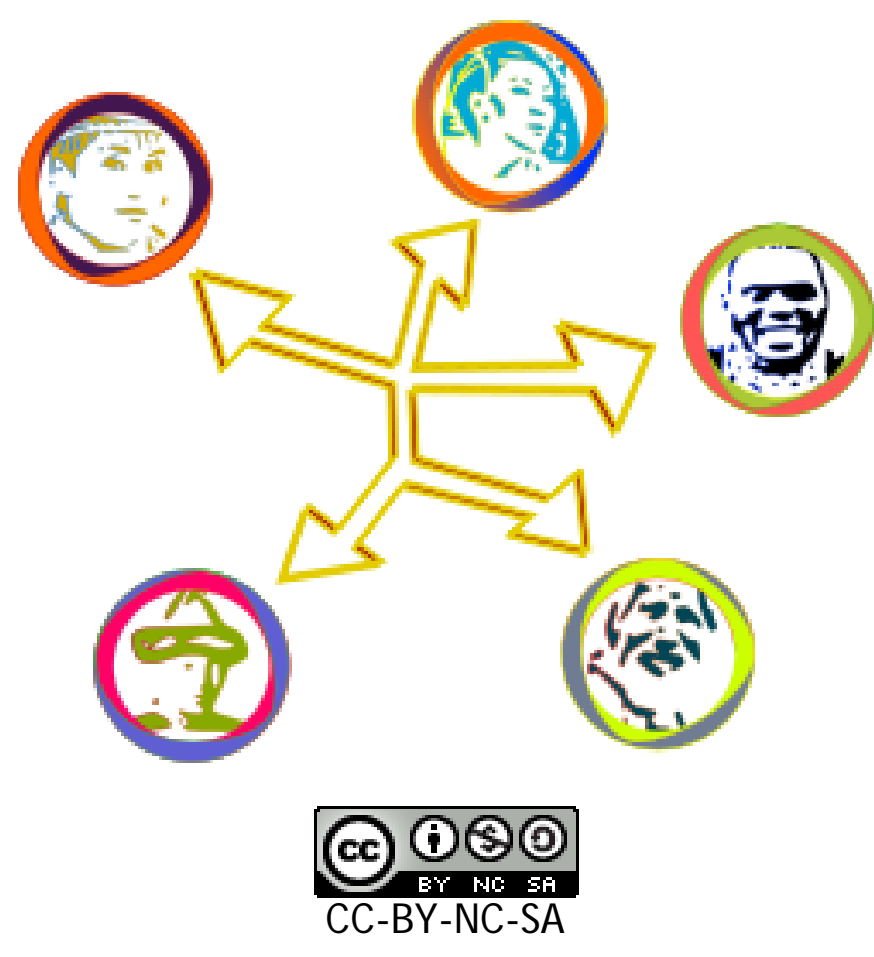

juin 2013 
Assurer une présence sur les réseaux sociaux est aujourd'hui incontournable pour les bibliothèques d'une certaine taille. Cette démarche permet de toucher un public étendu, d'assurer une médiation vers ses ressources et celles de ses partenaires, de développer la participation des usagers, et de montrer la connexion entre l'établissement et son quartier.

La bibliothèque de la Canopée souhaite assurer une forte présence sur les réseaux sociaux. Il en est de même pour la bibliothèque en préfiguration du Carré Saint-Lazare. Cette étude à un instant $\mathrm{T}$ doit servir de base pour déterminer quelles sont les plateformes sociales les plus intéressantes dans une bibliothèque de lecture publique. Les fonctionnalités, l'attrait de certains réseaux évoluant à la hausse ou à la baisse entraînera une réévaluation possible de ce benchmark.

\section{Les critères}

Afin de connaître les différents réseaux sociaux existant et leurs modalités d'utilisation nous avons retenu des critères d'analyse, selon la typologie suivante :

- les publics utilisateurs du réseau social : quels utilisateurs et quels usages ?

- $\quad$ public cible (âge, sexe, CSP...) et objectif initial (qui le réseau cible-t-il ?)

- communauté qui publie sur le réseau (entreprise, collectivité...)

- distinction personne/institution

- $\quad$ type de contenu (texte, photo, vidéo, musique...)

- $\quad$ usages réels du réseau et leur évolution (privé, perso, commercial...)

- évolution de la popularité

- évolution de la solution technique (évolution des fonctionnalités)

- la bibliothèque : quels usages ? Quelles possibilités offre une page institutionnelle ?

- $\quad$ offre de médiation aux usagers (conseil, valorisation, suggestion...)

- degré de participation des usagers aux actions de la bibliothèque (interaction entre usagers et avec la bibliothèque)

- identité de la bibliothèque (est-elle reconnaissable facilement ?)

- modalités de production (back office, ergonomie, facilité de prise en main)

- évaluation quantitative et qualitative (statistiques de fréquentation, de participation...)

- l'interaction entre les publics et la bibliothèque : quelles conditions d'utilisation du réseau social ? Quelle est l'image publique du réseau?

- nécessité et condition d'inscription (limite d'âge, indication de son nom...) 
- capacité à toucher les utilisateurs (visibilité des publications de la bibliothèque)

- incitation commerciale (acheter des options, publicité...)

- $\quad$ support mobile (application, format de lecture smartphone ou tablette)

- ergonomie (front office) et langue

- propriété des données (personnelles et le contenu)

- image médiatique (positive et négative dans les médias généralistes, spécialisés, professionnels)

- Pour terminer : quels sont les éléments marquants du réseau que nous identifions ?

- Usages intéressants ou pas (faits par une bibliothèque ou une autre entité)

Sur certaines plateformes, nous avons pu introduire un « critère tueur » : si un critère que nous désignons comme essentiel n'est pas rempli ou que l'on atteint un trop grand nombre de critères non réunis, inutile de poursuivre le benchmark et le réseau social en question est écarté.

\section{Les réseaux}

L'analyse fut menée sur 45 réseaux sociaux que nous avons listés et classés par similitudes d'usage/type de contenu afin de favoriser les comparaisons:

- les «facebook»

- Facebook

- Google +

- Friendster

- Tagged

- Bebo

- Netlog

- Skyrock

- $\mathrm{Hi5}$

- Peuplade (Paris)

- Les «twitter»

- Twitter

- Identi.ca

- Path

- Reddit 
- Pheed

- Partage d'image, photo et vidéo

- Instagram

- Flickr

- Youtube

- Dailymotion

- Vimeo

- Vine

- «Épinglage »

- Pinterest

- Tumblr

- Who Art You

- Pearltrees

- Musique

- Myspace

- Spotify

- Deezer

- Grooveshark

- Soundcloud

- Mupiz

- Music me

- Littérature

- Babelio

- Sens critique

- We love words

- riffle

- Les agrégateurs

- Diigo

- Delicio.us

- Netvibes 
- Hello coton

- Symbaloo

- Scoop.it

- Les inclassables

- Slideshare

- Glowbl

- Foursquare

- Paris.onvasortir.com

Cette sélection fit le choix d'inclure des sites qui ne sont pas à proprement parler des réseaux sociaux mais que nous avons souhaité tout de même analyser pour en connaître leurs modalités communautaires et de partage de contenu.

\section{La méthodologie}

Avant toute chose, il a fallu se créer un compte sur le réseau social analysé. Il n'est pas forcément aisé de découvrir toutes les fonctionnalités que propose un réseau social sans s'y inscrire. Il est donc conseillé d'explorer les possibilités qu'offre une page personnelle (back office et côté profil public d'utilisateur) et de naviguer sur des profils et pages d'institutions (front office : bibliothèque, marque, entreprise, association, collectivité...). Les usages d'un réseau pouvant être multiples, il est intéressant de confronter l'usage qu'en font différentes entités.

Pour compléter les différents critères, il est conseillé de mener les recherches sur le réseau social étudié « en vrac » et de remplir les évaluations $(++/+/-/--$ et commentaires) au fur et à mesure des informations trouvées. Par expérience, cette méthode prend moins de temps qu'une recherche méthodique et ciblée, critère par critère. Il est en effet plus complexe de trouver des informations sur un critère isolé. Pour autant, cette méthode au fil de l'eau nécessite de recouper les données: il ne faut pas hésiter à revenir sur un critère, affiner, modifier son évaluation et la nuancer. Cette démarche est d'autant plus pertinente qu'elle s'effectue par comparaison avec les autres réseaux étudiés.

L'évaluation des critères, lorsqu'elle est quantifiable, se traduit par les indicateurs suivants : $++/+/-/--$. Seuls 15 critères sont à quantifier. Cette catégorisation permet de noter le réseau social sur 30 points. A cela s'ajoute un code couleur qui donne une impression visuelle générale positive ou négative : vert pour positif $(++$ et +$)$, rouge pour négatif (- et --).

Enfin, il ne faut pas hésiter à conserver dans un fichier les sources utiles et intéressantes : elles pourront être reconsultées plus tard pour une étude approfondie de certains réseaux sociaux choisis pour la bibliothèque. 


\section{Classement des plateformes par genre}

\begin{tabular}{|c|c|}
\hline Facebook-like & Note sur 30 \\
\hline$\underline{\text { facebook }}$ & 19 \\
\hline$\underline{\text { Google }+}$ & 24 \\
\hline$\underline{\text { Friendster }}$ & rejeté \\
\hline$\underline{\text { tagged }}$ & rejeté \\
\hline$\underline{\text { bebo }}$ & rejeté \\
\hline netlog & rejeté \\
\hline$\underline{\text { skyrock }}$ & rejeté \\
\hline$\underline{\text { Hi5 }}$ & rejeté \\
\hline$\underline{\text { Peuplade }}$ & -6 \\
\hline
\end{tabular}

Parmi les dix réseaux testés seuls trois ont retenu notre attention :

- l'inévitable Facebook pour ses possibilités de réseautage et son utilisation démocratisée

- le jeune Google+ pour ses capacités à toucher un public complémentaire de celui de Facebook

- le tout petit Peuplade pour son réseau à échelle humaine et ses contacts de proximité potentiels

Toutefois, Peuplade n'est plus modéré depuis 2010, et continue de vivre par ses membres actifs. On attendra tout de même de voir son évolution d'ici fin 2014 pour s'y engager.

Ont été écartés d'office car hors sujet: Tagged, Badoo, Hi5, réseaux positionnés sur le créneau site de rencontres et Friendster car le réseau s'est repositionné comme site de jeu social. Bebo lui est malheureusement impraticable sur notre parc informatique, et Netlog est à l'abandon. Skyblog aurait pu présenter un intérêt mais même si l'offre de médiation et le public sont là, la présence d'une médiathèque sur le réseau semble être à l'opposé des préoccupations des membres de celui-ci. 


\begin{tabular}{|c|c|}
\hline Twitter-like & Note sur 30 \\
\hline$\underline{\text { Twitter }}$ & 18 \\
\hline$\underline{\text { Identi.ca }}$ & 0 évolution en cours \\
\hline$\underline{\text { Path }}$ & Rejeté \\
\hline$\underline{\text { reddit }}$ & Rejeté \\
\hline$\underline{\text { Pheed }}$ & 21 \\
\hline
\end{tabular}

Twitter reste l'outil incontournable des plateformes de blogging rapide et le seul qui se détache vraiment en 2013. Il demande cependant un fort investissement qui doit être pris en compte lorsqu'une bibliothèque souhaite s'y investir. Identi.ca n'est utilisé que par une communauté très restreinte et ses nouvelles performances doivent être encore évaluées. Pheed s'avère être une plateforme particulièrement complète, innovante et prometteuse. Reste à attendre sa version française.

Path et Reddit ont été éliminés pour des raisons diverses : nombre limité de contacts pour Path et multiplication de critères négatifs pour Reddit.

\begin{tabular}{|c|c|}
\hline Partage de photos et de vidéo & Note sur 30 \\
\hline$\underline{\text { instagram }}$ & 16 \\
\hline$\underline{\text { flickr }}$ & 14 \\
\hline$\underline{\text { youtube }}$ & 16 \\
\hline$\underline{\text { dailymotion }}$ & 20 \\
\hline$\underline{\text { vimeo }}$ & 17 \\
\hline$\underline{\text { vine }}$ & \\
\hline
\end{tabular}

Les sites et applications de retouche et partage de photos étudiés, présentent des usages complémentaires. Instagram est une application mobile et Flickr, si la partie mobile tend à être de plus en plus développée, reste un outil majoritairement utilisé en ligne pour la création et le partage d'albums photos. Instagram serait donc préconisé pour un usage mobile et créatif avec l'utilisation des filtres quand Flickr serait plus adapté à l'animation de communautés en ligne sur le long terme.

Il en va de même pour les sites de partage vidéo. Si les deux plus importantes plateformes étudiées, Youtube et Dailymotion, semblent être incontournables en matière de popularité et de fonctionnalités étendues (en ligne comme sur mobile). Ils présentent toutefois des 
inconvénients de taille comme le format publicitaire «Instream » et l'énorme masse de contenus disponibles (Dailymotion restant supérieur à Youtube sur les fonctions de personnalisation).

Vimeo peut donc apparaître comme une alternative intéressante pour des contenus à forte valeur ajoutée, mis en valeur par l'esthétique du site et son image de communauté créative. De la même manière qu'Instagram peut être utilisé en complémentarité avec Flickr, l'usage de Vimeo n'exclut pas celui de Vine, une application mobile relativement récente mais à suivre, notamment pour son potentiel créatif.

\begin{tabular}{|c|c|}
\hline Musique & Note sur 30 \\
\hline Myspace & 16 \\
\hline$\underline{\text { spotify }}$ & rejeté \\
\hline deezer & 17 \\
\hline grooveshark & 25 \\
\hline soundcloud & rejeté \\
\hline mupiz & 9 \\
\hline Music Me & \\
\hline
\end{tabular}

Trois plateformes musicales sont à retenir : Soundcloud, Grooveshark et Spotify. Si la qualité du catalogue est l'atout de Spotify, elle est justement le point faible de Grooveshark (doublons). Pour autant, Grooveshark offre davantage de fonctionnalités d'interaction entre les utilisateurs et de médiation. Soundcloud se révèle être une plateforme originale par ses contenus (créations originales de DJ, mashup...) mais sa gestion des droits est mouvante et la version française n'existe pas encore.

Chacun de ces trois sites suggère donc des usages particuliers : Spotify pour la création de playlists, Grooveshark pour le partage de musique et Soundcloud pour la découverte de nouveaux titres et artistes.

Music Me obtient une note moyenne car malgré ses qualités, le site envisage uniquement des interactions via d'autres réseaux sociaux (partage sur Facebook).

Enfin, trois plateformes ont été écartées: Myspace à cause de son obsolescence avérée, Deezer pour son incompatibilité avec le réseau Ville de Paris et Mupiz qui ne correspond pas à nos attentes en étant exclusivement un site pour les musiciens. 


\begin{tabular}{|c|c|}
\hline «Epinglage » et curation & Note sur 30 \\
\hline pinterest & 8 \\
\hline$\underline{\text { tumblr }}$ & -2 \\
\hline$\underline{\text { Who art'you }}$ & 0 \\
\hline pearltrees & 20 \\
\hline$\underline{\text { Scoop.it }}$ & \\
\hline
\end{tabular}

La « curation » est le moyen de faire ressortir rapidement des informations, photographies, vidéos, et de les connecter entre elles. De telles plateformes se distinguent des agrégateurs par leur côté plus dynamique, convivial et des possibilités supérieures de partage.

Tumblr, réseau en développement accéléré se distingue rapidement du lot car il mélange des fonctions de micro-blogging à une interface conviviale et jouit d'une image à la fois dynamique, décalée et élitiste.

Scoop.it séduit par sa facilité d'utilisation ainsi que par ses fonctionnalités ouvertes vers nombre de réseaux sociaux. Il présente également un grand intérêt en tant qu'outil de veille documentaire.

Pearltrees déçoit par contre, notamment sur l'aspect de la convivialité et de la recherche de partenaires, tout comme Pinterest sur les fonctions de partage. Who art'you est encore trop récent doit se développer et coûter moins cher aux utilisateurs.

\begin{tabular}{|c|c|}
\hline Littérature & Note sur 30 \\
\hline$\underline{\text { Babelio }}$ & 2 \\
\hline$\underline{\text { We love words }}$ & -13 \\
\hline$\underline{\text { Sens critique }}$ & 8 \\
\hline$\underline{\text { riffle }}$ & -23 \\
\hline
\end{tabular}

Quatre réseaux sociaux «littéraires» ont été étudiés: Babelio, Sens critique, We love words et Riffle. We love words et Riffle ne semblent pas convenir à l'usage d'une bibliothèque car trop pointus ou trop loin du public français.

Babelio possède un grand fonds de référence en littérature et permet de mettre en ligne des analyses, de commenter des textes de créer des listes de coups de cœur ou de nouveautés par exemple. Mais attention, une page désertée ou insuffisamment mise à jour donne l'impression d'une bibliothèque moribonde. 
Sens critique est une interface multimédia, plus jeune et plus ergonomique. Bien qu'il possède moins de références littéraires c'est une mine pour les autres supports : films, jeux vidéo. Ce réseau colle également à l'actualité. Il permet aussi de créer des listes et de publier des critiques.

\begin{tabular}{|c|c|}
\hline Les agrégateurs & Note sur 30 \\
\hline$\underline{\text { diigo }}$ & 8 \\
\hline$\underline{\text { Delicio.us }}$ & 8 \\
\hline$\underline{\text { netvibes }}$ & rejeté \\
\hline Hello coton & 9 \\
\hline$\underline{\text { symbaloo }}$ & \\
\hline
\end{tabular}

Cinq outils composaient cette étude sur les agrégateurs. Diigo et Delicious, sites de sauvegarde et de partage de favoris en ligne à vocation sociale sont relativement connus. Ils comptent beaucoup d'utilisateurs à travers le monde mais n'ont pas retenu notre attention malgré des fonctionnalités intéressantes. Leur utilisation et leur navigation sont peu aisées et nécessitent une bonne prise en main. Nous avons préféré Netvibes et notamment Symbaloo, à l'interface graphique plus colorée et ludique et mêlant fils RSS et sites web. Son utilisation en bibliothèque, couplée à un outil comme Facebook ou Twitter pourrait être intéressante.

Hello Coton, site qui regroupe des articles de blogs pour et par un public féminin, a très vite été écarté.

\begin{tabular}{|c|c|}
\hline Les inclassables & Note sur 30 \\
\hline$\underline{\text { slideshare }}$ & 5 \\
\hline glowbl & 1 \\
\hline$\underline{\text { foursquare }}$ & 4 \\
\hline Paris.onvasortir.com & rejeté \\
\hline
\end{tabular}

Cette dernière catégorie un peu fourre-tout rassemblait des réseaux aux fonctions très variés, depuis le partage de présentations «powerpoint» comme slideshare à une application de géolocalisation comme Foursquare. Chaque réseau présente ses avantages et inconvénients pour ces usages spécifiques. Foursquare a particulièrement retenu notre attention dans une optique de fidélisation d'un type de public, toutefois la popularité de cette plateforme tend à s'essouffler après un départ en fanfare. 


\section{Classement des plateformes par note sur 30}

TOP 10

\begin{tabular}{|c|c|}
\hline Plateforme & Note sur 30 \\
\hline Google + & 25 \\
\hline Soundcloud & 21 \\
\hline Pheed & 20 \\
\hline Vimeo & 20 \\
\hline Scoop.it & 19 \\
\hline Facebook & 18 \\
\hline Twitter & 17 \\
\hline Vine & 17 \\
\hline Grooveshark & 16 \\
\hline Dailymotion & \\
\hline
\end{tabular}

5 plateformes à surveiller

\begin{tabular}{|c|c|}
\hline Plateforme & Note sur 30 \\
\hline Foursquare & 4 \\
\hline Glowbl & 1 \\
\hline Peuplade & -6 \\
\hline Pinterest & 8 \\
\hline Who art'you & -2 \\
\hline
\end{tabular}




\begin{tabular}{|c|c|c|c|c|}
\hline \multirow{2}{*}{\begin{tabular}{c|c|} 
RS \\
Facebook
\end{tabular}} & & Critères & Evaluation & Commentaires \\
\hline & \multirow[t]{7}{*}{ Publics } & cible & particuliers et professionnels & tout le monde, en partulier les "jeunes" (mois de 35 ans) et les professionnels. \\
\hline & & communauté qui publie & tout le monde & particulier, institution publique, entreprise privée, marque, groupe de personnes (musique, danse...) etc. \\
\hline & & distinction personne/institution & ++ & personne=profil/ institution=page (like). Peuvent être aussi créés: groupe (commmunauté d'usagers) et évènement (annonce un RDV). \\
\hline & & type de contenu & multiple & texte (article, statut, info pratique, événement...), photo, vidéo, liens \\
\hline & & usages & perso et pro, privé et public & $\begin{array}{l}\text { avec l'arrivée des parents, des profs, des recruteurs sur FB, les jeunes I'utilisent comme la face officielle de leur vie (vrai nom...). } \\
\text { Augmentation de l'usage mobile. }\end{array}$ \\
\hline & & $\begin{array}{l}\text { évolution de la popularité (date de } \\
\text { création, nb d'utilisateurs) }\end{array}$ & $\begin{array}{l}\text { RS le + utilisé en France, en } \\
\text { augmentation mais en recul chez } \\
\text { les ados ++ }\end{array}$ & $\begin{array}{l}\text { lancement en 2004, ouvert au public en 2006. 1,06 milliard d'utilisateurs dans le monde dont } 26 \text { millions de Français. Augmentation des } \\
\text { seniors mais baisse des ados (au profit d'autres RS). }\end{array}$ \\
\hline & & évolution technique & ++ & $\begin{array}{l}\text { solution fiable, FB développe chaque année de nouvelles applications (jeu, messagerie...) / MAIS les utilisateurs n'en sont pas prévenus } \\
\text { (apparition ET disparition d'appli) }\end{array}$ \\
\hline & \multirow[t]{5}{*}{ Bibliothèque } & offre de médiation & ++ & $\begin{array}{l}\text { communiquer et valoriser (un événement, une nouveauté, une thématique), décrire (un document, une expo, une animation), suggérer } \\
\text { (en "aimant" d'autres pages), inviter (à un événement), discuter / écrire un article: la fonction est peu développée et présente peu } \\
\text { d'intérêt -> privilégier le lien vers article de blog }\end{array}$ \\
\hline & & participation des usagers & ++ & $\begin{array}{l}\text { possibilité de publier un message public, liker, commenter, partager, recommander, écrire un message privé, suggérer une modification, } \\
\text { discuter (messagerie instantanée, appel vidéo) }\end{array}$ \\
\hline & & identité de la bibliothèque & ++ & page personnalisable: photos, ajout d'applications (ex: page BPI et ses podcasts ou Bibliosesame), infos pratiques \\
\hline & & back office & ++ & facilité d'utilisation, intuitif. Même présentation que pour le front office. \\
\hline & & évaluation quantitative et qualitative & ++ & stat détaillées: performance de la page et engagement des fans, informations sur les fans touchés, viralité des publications... \\
\hline & \multirow{7}{*}{ Interaction } & nécessité et condition d'inscription & + & inscription obligatoire pour publier et interagir mais pas pour voir la page, inscription à partir de 13 ans \\
\hline & & capacité à toucher les utilisateurs & - & $\begin{array}{l}\text { FB ne diffuse les post qu'à environ } 15 \% \text { de ses "amis", principe de popularité d'un page: moins un utilisateur consulte une page, moins les } \\
\text { post de celle-ci vont s'afficher sur la timeline de l'usager. }\end{array}$ \\
\hline & & incitation commerciale & - & $\begin{array}{l}\text { publicité ciblée dans la navigation profil mais pas sur les pages, incitation à payer pour la promotion d'un post (et contrer le principe de } \\
\text { popularité). }\end{array}$ \\
\hline & & front office, ergonomie + langue & ++ & $\begin{array}{l}\text { fil d'actualité avec une présentation chronologique des posts (présentation du post varie selon le contenu) + classement par contenu pour } \\
\text { aller directement aux photos/vidéos/événements + possibilité d'affichage par filtre (publication de la page/d'autres personnes sur la } \\
\text { page). Affichage clair et unique pour toutes les pages, importance du visuel iconographique. LANGUE: Français }\end{array}$ \\
\hline & & support mobile & oui ++ & appli android, apple, pour smartphone et tablette \\
\hline & & propriété des données & le réseau est propriétaire - & $\begin{array}{l}\text { l'utilisateur accord à FB "une licence non-exclusive, transférable, sous-licenciable, sans redevance et mondiale pour l'utilisation des } \\
\text { contenus de propriété intellectuelle que vous publiez sur Facebook ou en relation avec Facebook" (source: Facebook, Déclaration des } \\
\text { droits et responsabilités). Mais contrôle de la publicité des données avec les conditions de confidentialité à moduler }\end{array}$ \\
\hline & & $\begin{array}{l}\text { image médiatique (médias } \\
\text { généralistes et spécialisés) }\end{array}$ & + & $\begin{array}{l}\text { image positive et fiable: les médias généralistes ont leur page FB, parle du réseau comme un vecteur d'information et d'échange } \\
\text { (phénomène de buzz), publient les stats d'utilisation. les médias spécialisés analysent les stats d'utilisation, parlent des nouvelles } \\
\text { fonctionnalités. / image négative: cession des données à Facebook, traçage des utilisateurs et profilage. }\end{array}$ \\
\hline & \multicolumn{3}{|c|}{ usages intéressants ou pas } & $\begin{array}{l}\text { British Library: proposer du contenu et de l'info autre que ce qui concerne strictement la bib (photo du jour, énigme à résoudre, partage de } \\
\text { liens, articles, docs numérisés, expo...) = très bien, animation de communautés. La BPI intègre Bibliosesame à sa page: on peut poser une } \\
\text { question directement sur Facebook. }\end{array}$ \\
\hline
\end{tabular}

\begin{tabular}{|c|c|}
\hline Critères quantifiables remplis & $10++/ 30$ \\
$2+/ 30$ \\
$3-/ 30$ \\
\hline
\end{tabular}

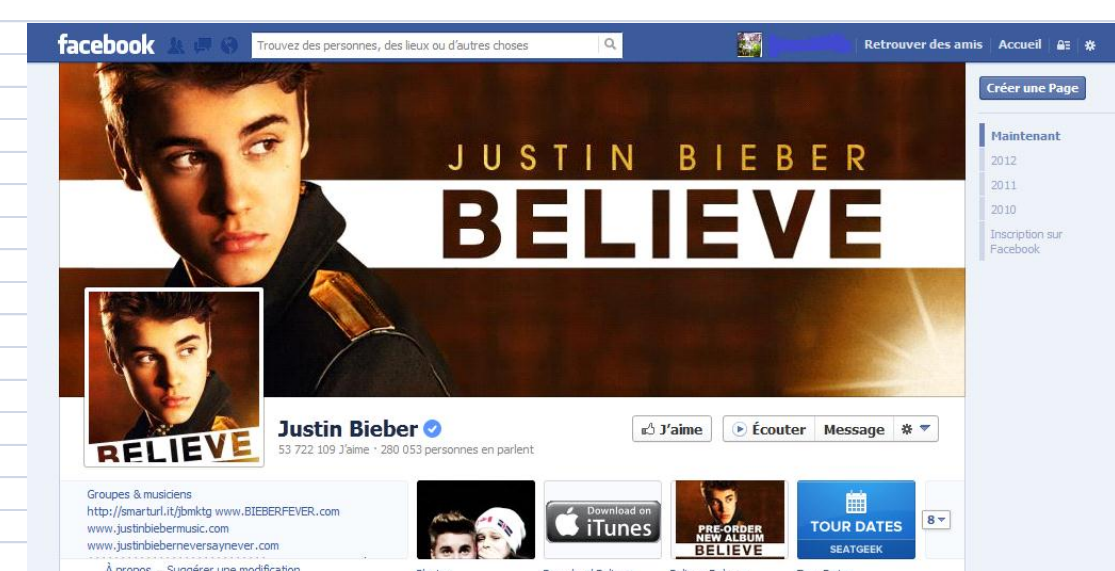




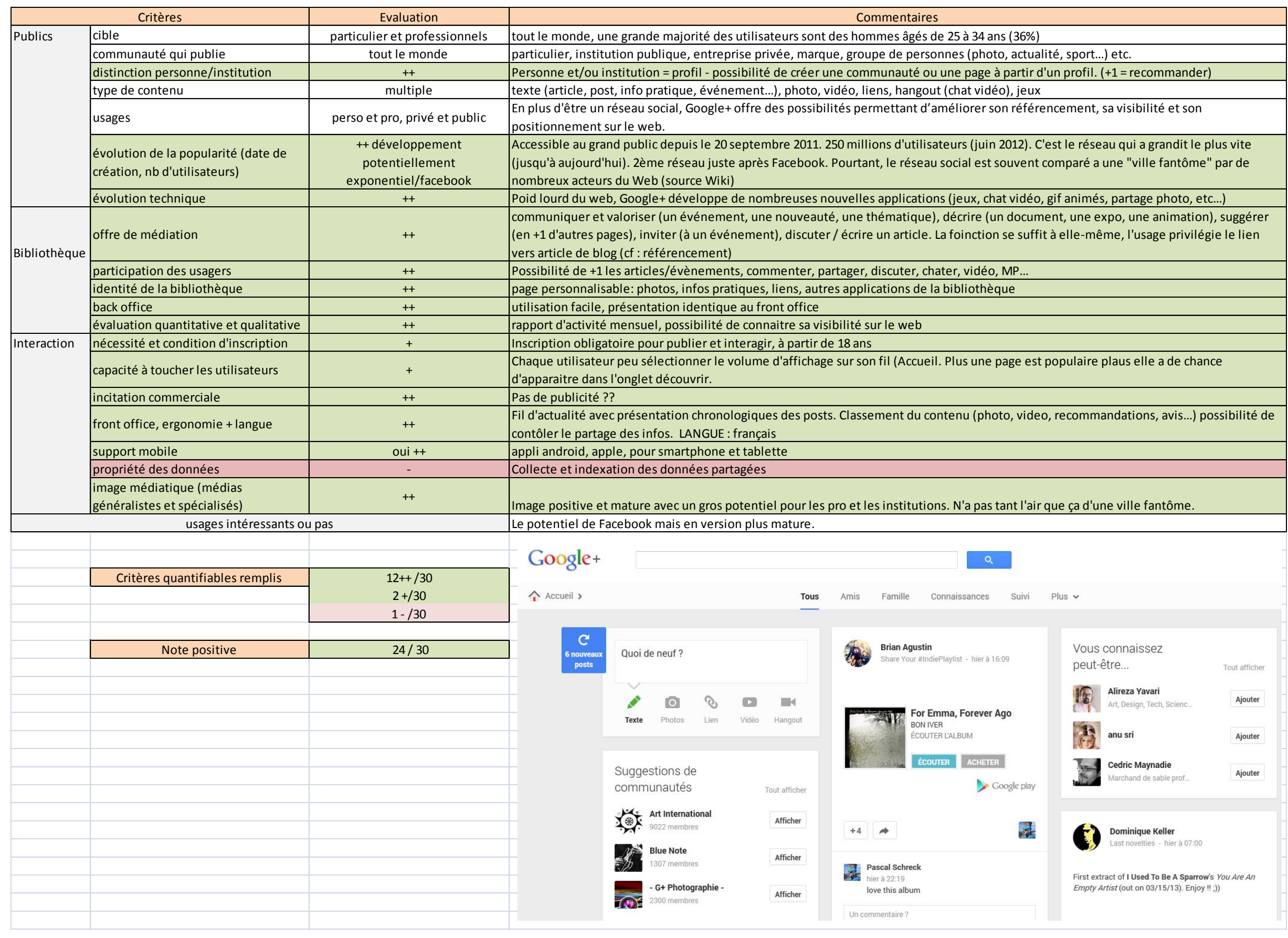




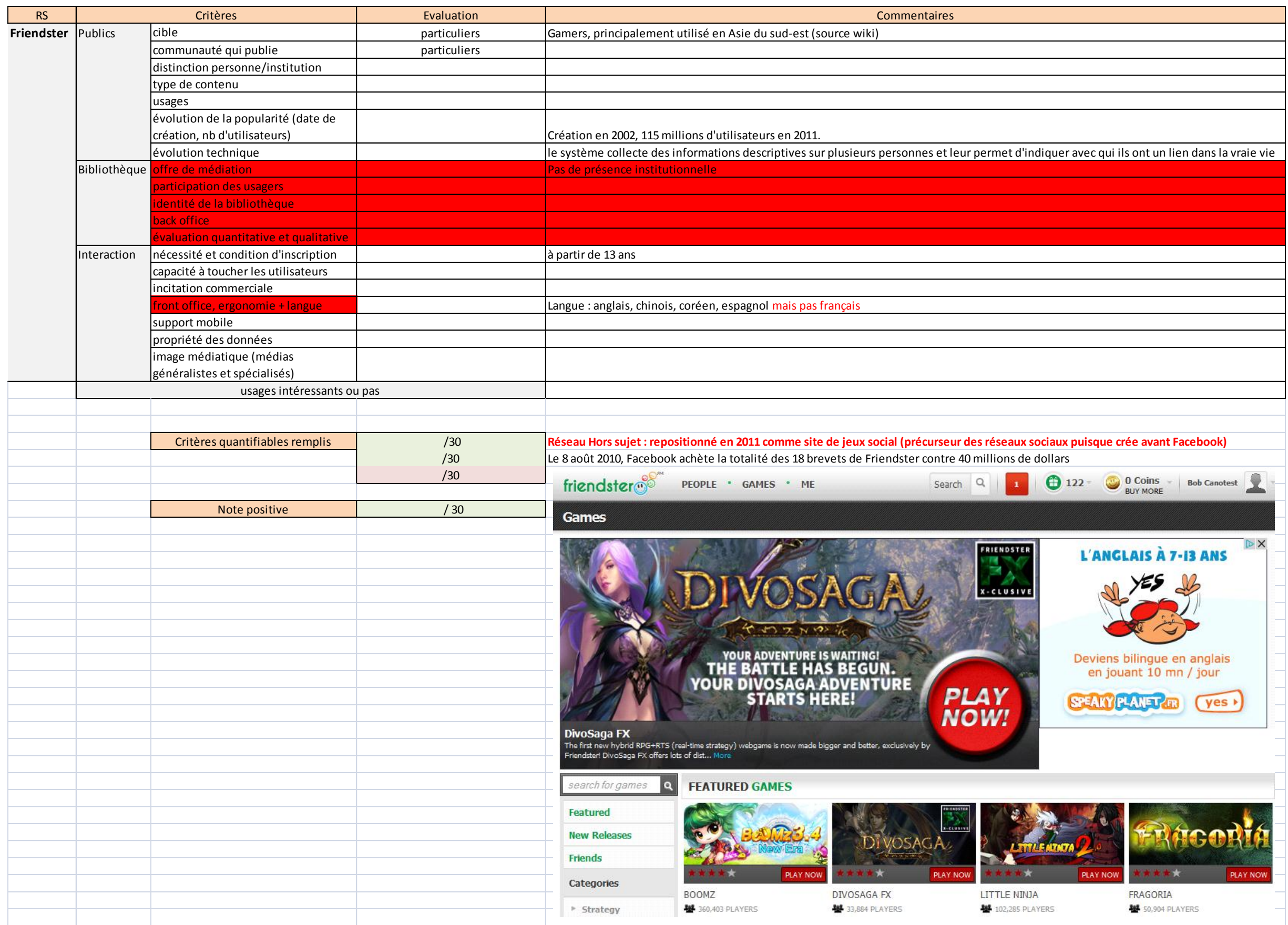




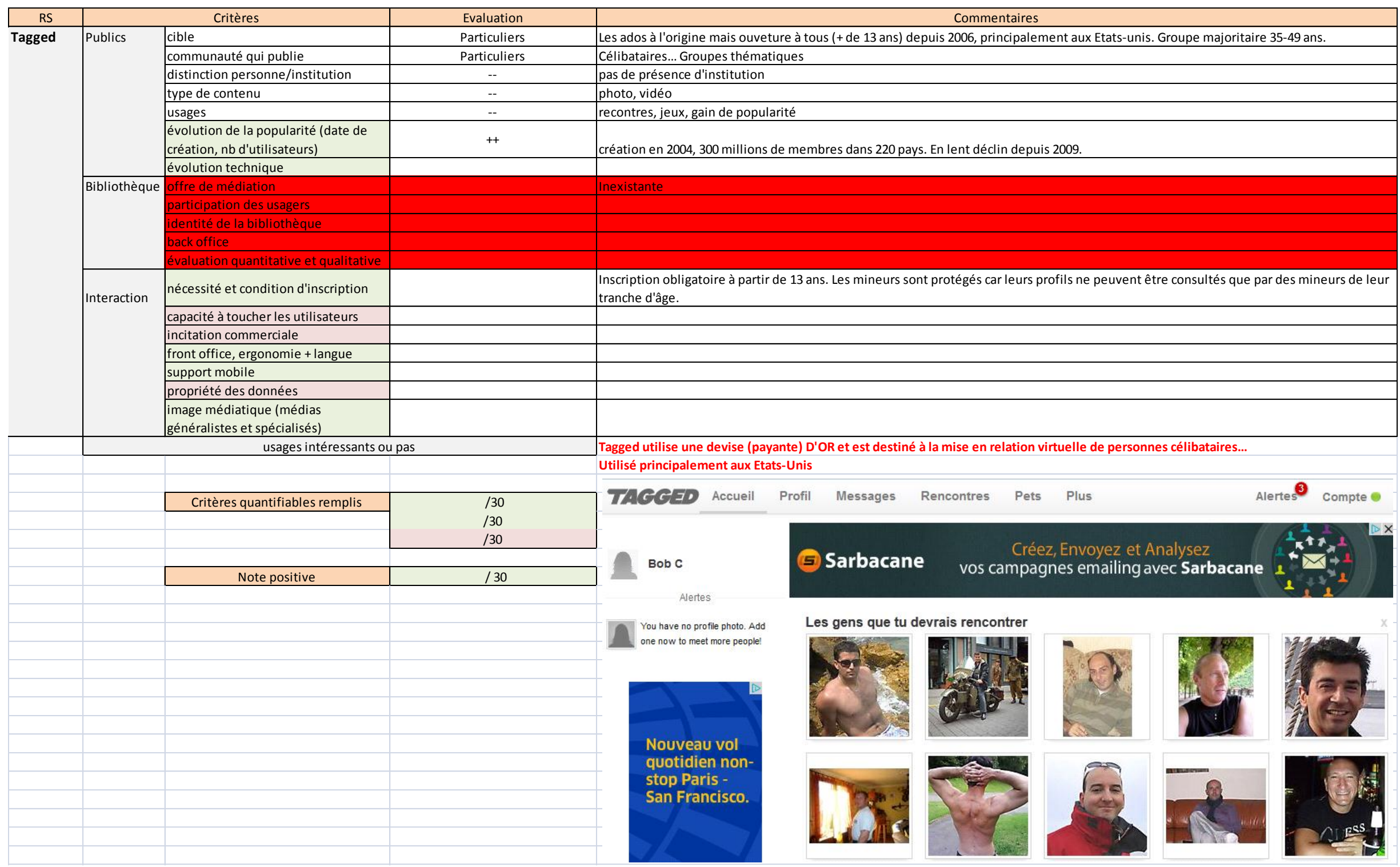




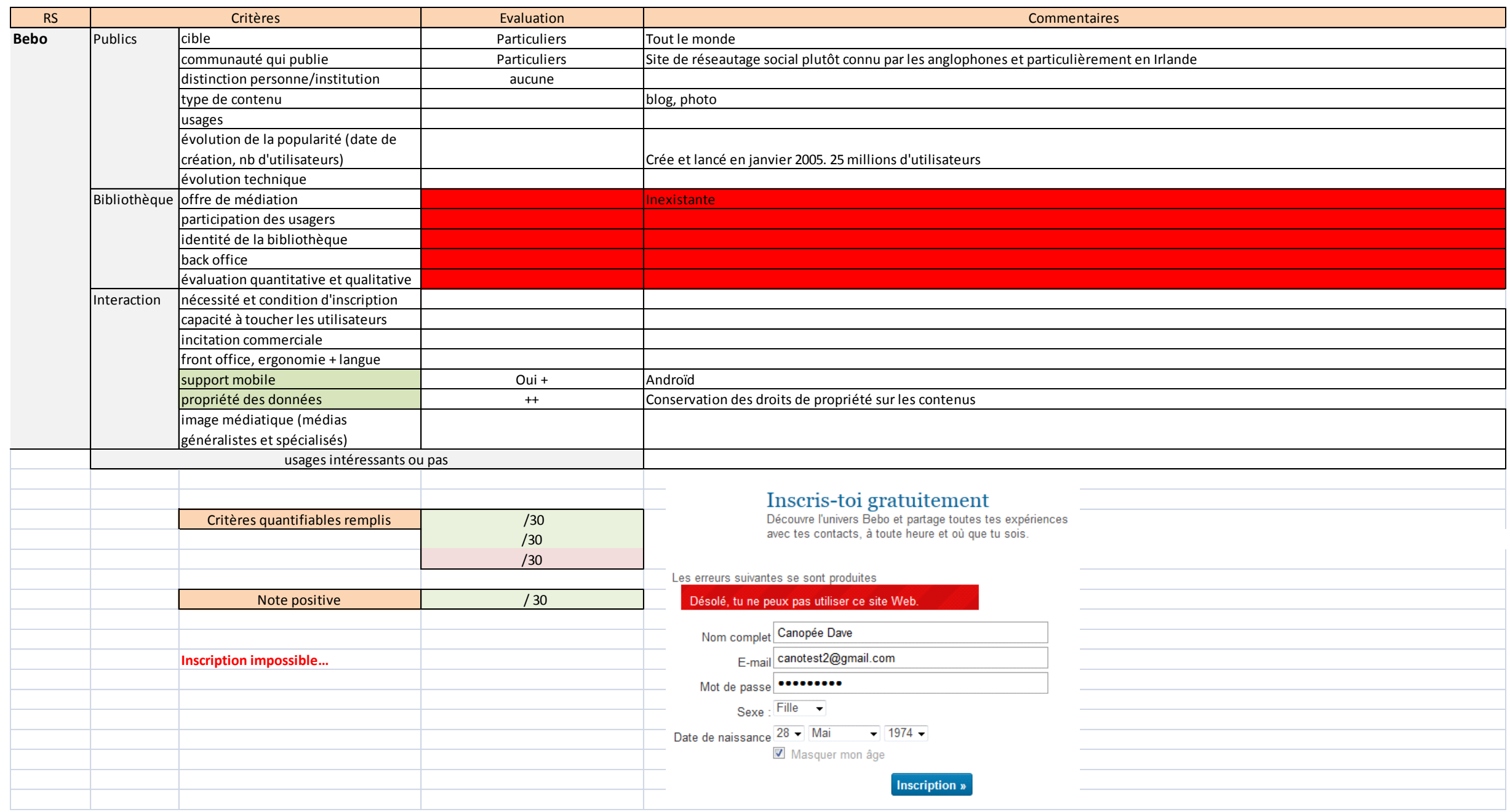




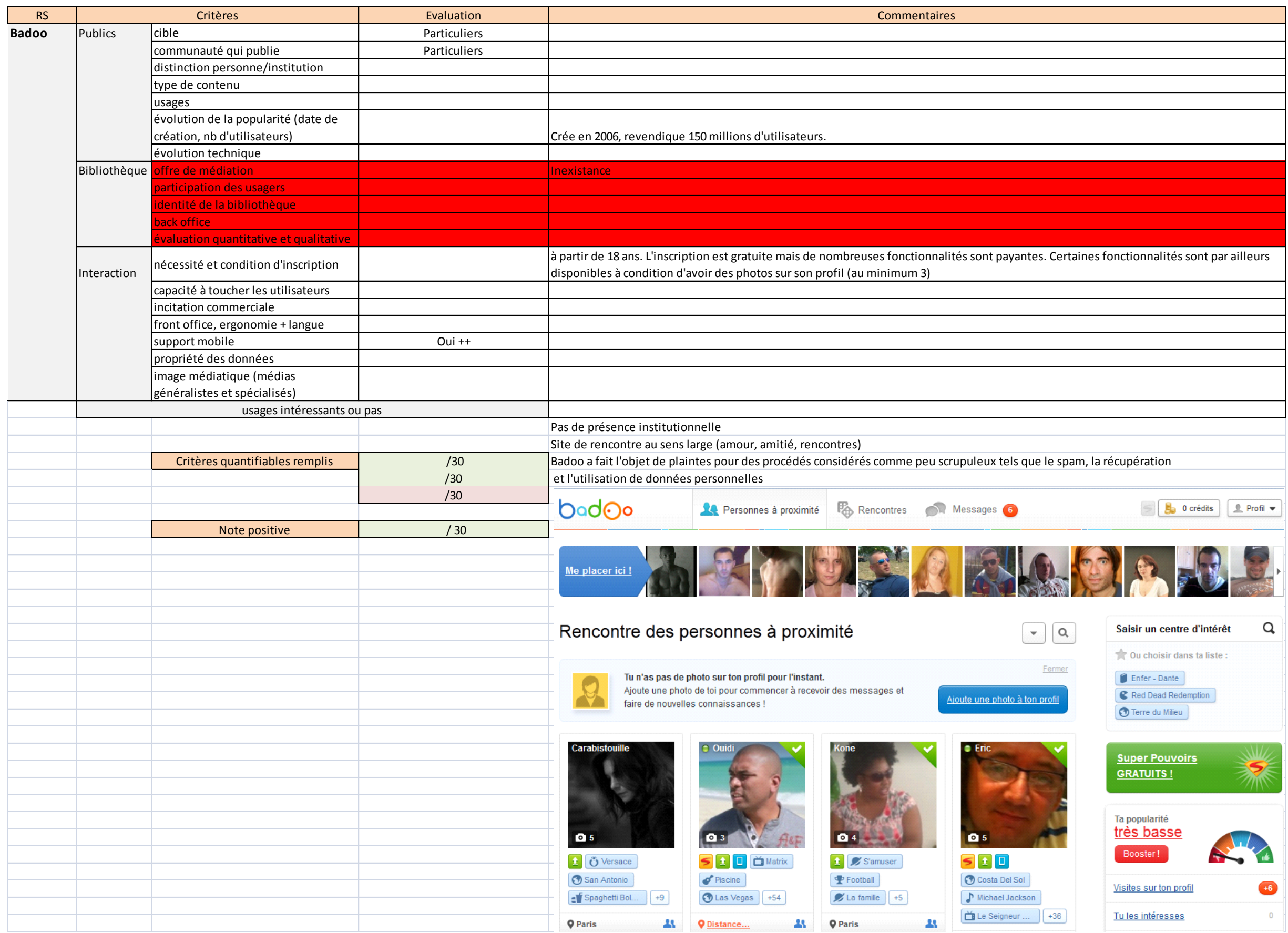




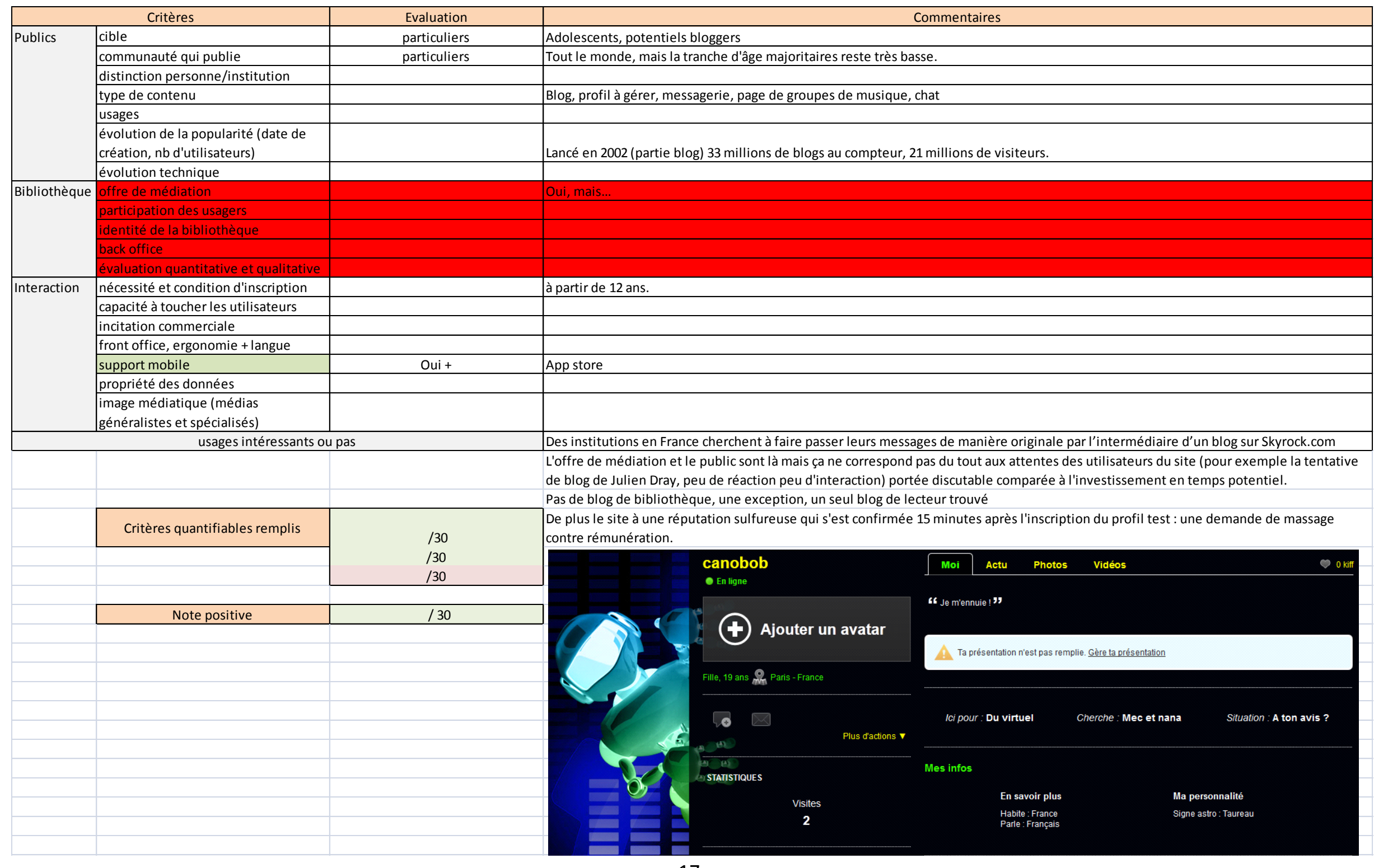

17 


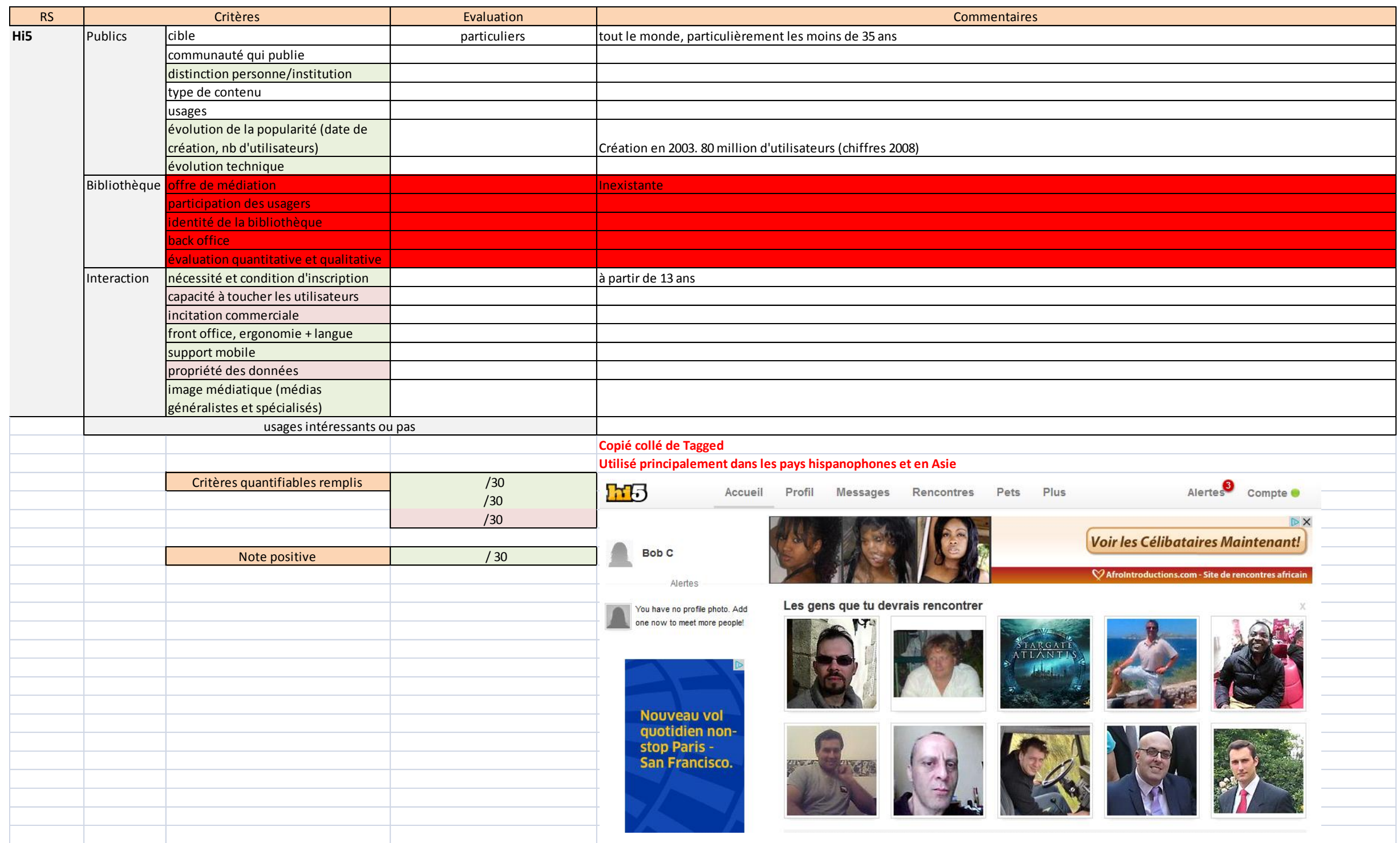




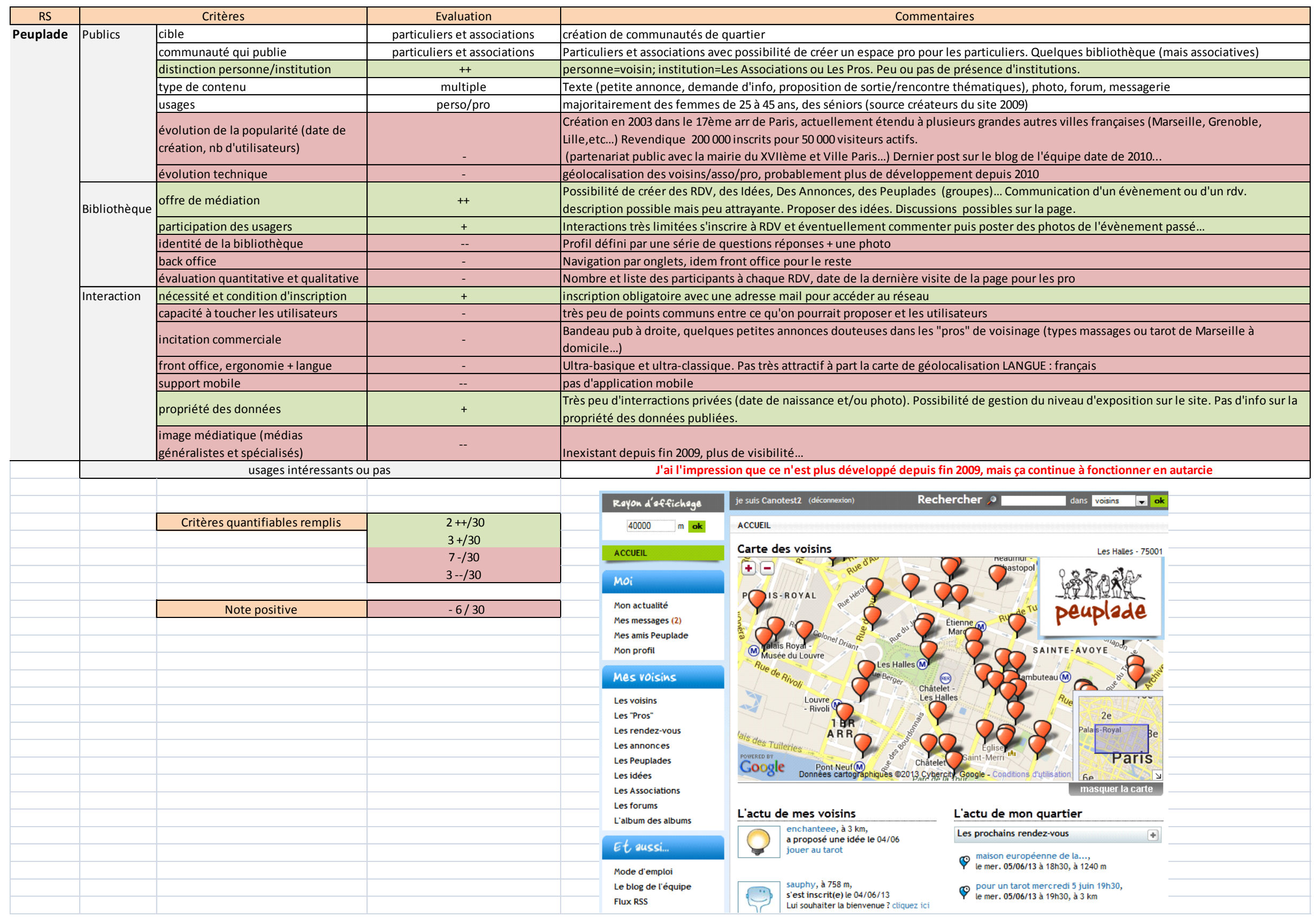




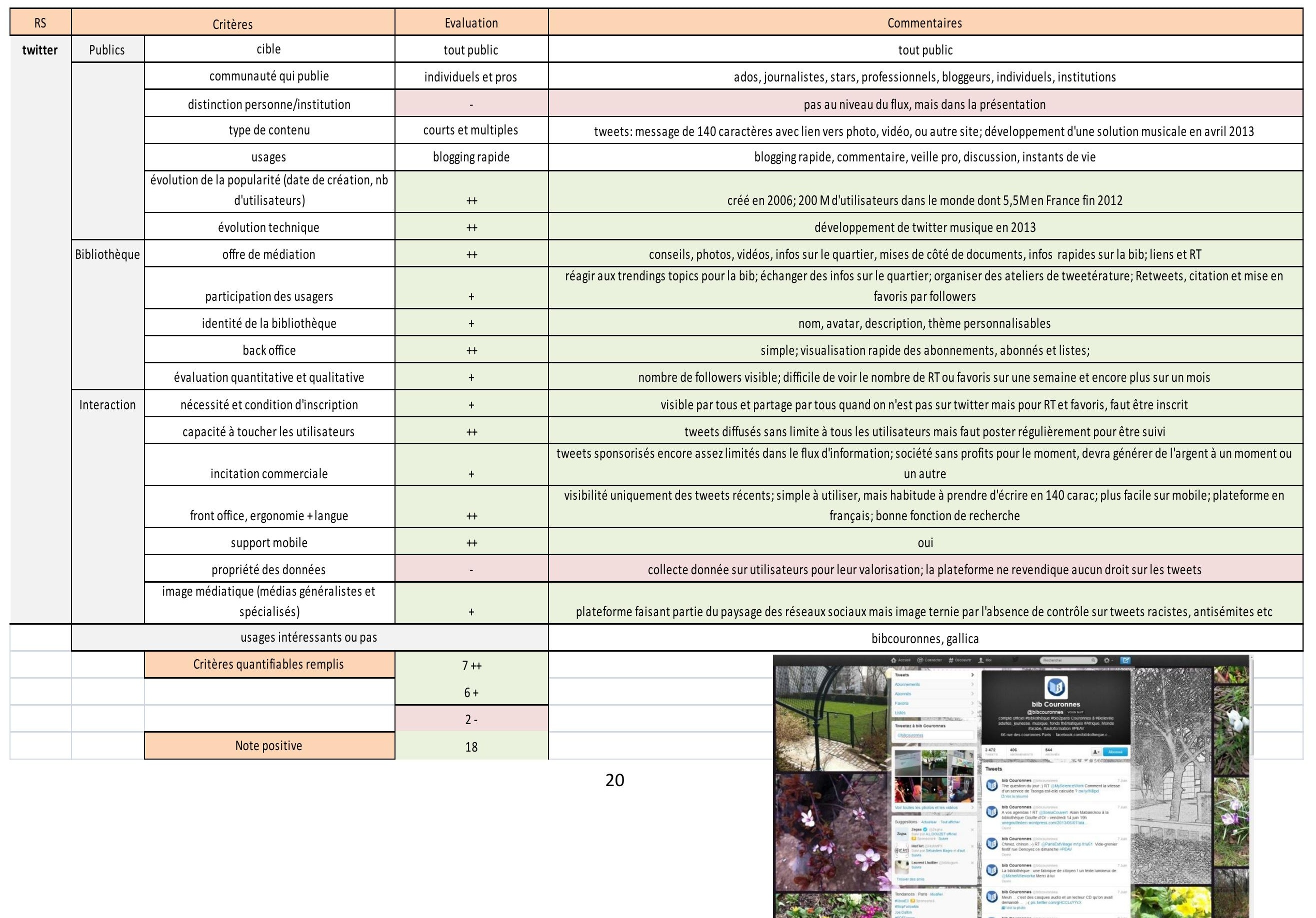




\begin{tabular}{|c|c|c|c|c|c|c|}
\hline RS & \multicolumn{2}{|r|}{ Critères } & Evaluation & \multicolumn{3}{|c|}{ Commentaires } \\
\hline \multirow[t]{19}{*}{ identi.ca } & Publics & cible & particuliers et organisations & \multicolumn{3}{|c|}{ tout public } \\
\hline & & communauté qui publie & individuels et pros & \multicolumn{3}{|c|}{ geeks et "libristes", adeptes des logiciels libres } \\
\hline & & distinction personne/institution & - & \multicolumn{3}{|c|}{ pas au niveau du flux, mais dans la présentation } \\
\hline & & type de contenu & courts et multiples & \multicolumn{3}{|c|}{140 caractères mais apparemment il existe des solutions techniques pour écrire en 320 caractères } \\
\hline & & usages & blogging rapide & \multicolumn{3}{|c|}{ blogging rapide, commentaire, veille pro, discussion, instants de vie } \\
\hline & & $\begin{array}{c}\text { évolution de la popularité (date de création, nb } \\
\text { d'utilisateurs) }\end{array}$ & - & \multicolumn{3}{|c|}{ 1,4 M de comptes; 100000 actifs sur 12 derniers mois et peut-être seulement 25 à 50000 vraies personnes } \\
\hline & & évolution technique & & \multicolumn{3}{|c|}{$\begin{array}{l}\text { des changements techniques le 1er juin 2013, notamment pour réduire le nombre de spammeurs et changer la solution libre de microblogging } \\
\qquad \text { (passage de status.net à pump.io) http://status.net/2013/01/09/preview-of-changes-to-identi-ca }\end{array}$} \\
\hline & \multirow[t]{5}{*}{ Bibliothèque } & offre de médiation & + & \multicolumn{3}{|c|}{ conseils, photos, vidéos, infos sur le quartier, mises de côté de documents, infos rapides sur la bib; liens } \\
\hline & & participation des usagers & & & & \\
\hline & & identité de la bibliothèque & + & \multicolumn{3}{|c|}{ nom, avatar, description } \\
\hline & & back office & & & & \\
\hline & & évaluation quantitative et qualitative & & & & \\
\hline & \multirow[t]{7}{*}{ Interaction } & nécessité et condition d'inscription & & & & \\
\hline & & capacité à toucher les utilisateurs & & & & \\
\hline & & incitation commerciale & & & & \\
\hline & & front office, ergonomie + langue & - & \multicolumn{3}{|c|}{$\begin{array}{l}\text { austère; disponible en français; impossibilité de s'inscrire avant le changement de solution technique; possibilité de lier les comptes twitter et } \\
\text { identi.ca }\end{array}$} \\
\hline & & support mobile & & & & \\
\hline & & propriété des données & + & \multicolumn{3}{|c|}{ données restent propriété de l'utilisateur; peut les retirer en clôturant son compte; pas de revente d'infos sur les utilisateurs } \\
\hline & & $\begin{array}{l}\text { image médiatique (médias généralistes et } \\
\text { spécialisés) }\end{array}$ & + & \multicolumn{3}{|c|}{ dans média spécialisés, vu comme alternative libre à twitter; inconnu par ailleurs } \\
\hline & \multicolumn{3}{|c|}{ usages intéressants ou pas } & \multicolumn{3}{|c|}{$\begin{array}{l}\text { bib de St Palais est sur identi.ca; http://identi.ca/mia64; http://thisisabore.net/post/2010/09/16/De-l-int\%C3\%A9r\%C3\%AAt-d-Identi.ca-et- } \\
\text { Status.net-face-\%C3\%A0-Twitter }\end{array}$} \\
\hline & & Critères quantifiables remplis & & \multirow{5}{*}{\multicolumn{2}{|c|}{ 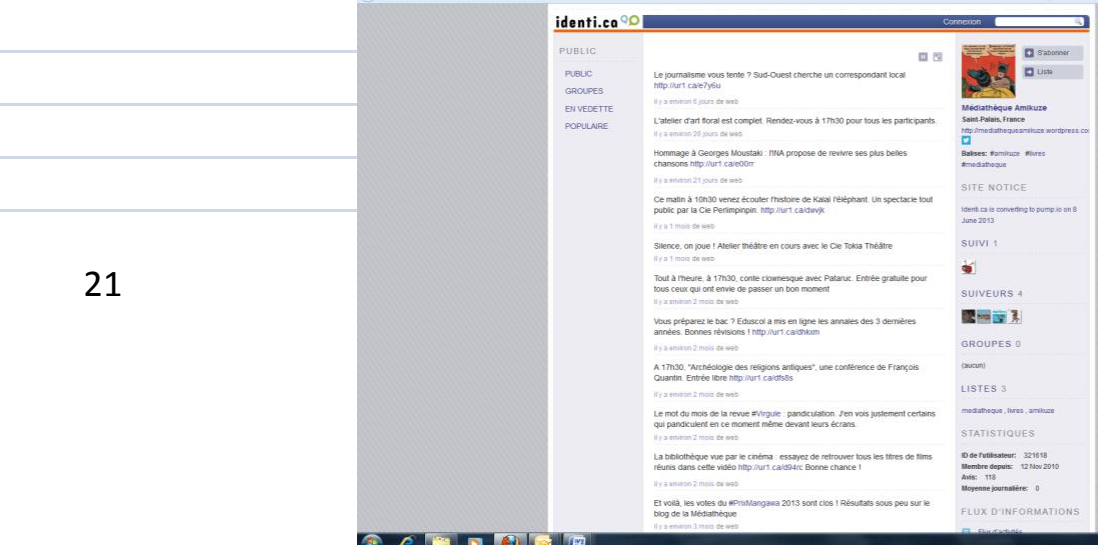 }} & \\
\hline & & & & & & \\
\hline & & & & & & \\
\hline & & Note positive & 0 & & & \\
\hline & & & & & & \\
\hline
\end{tabular}




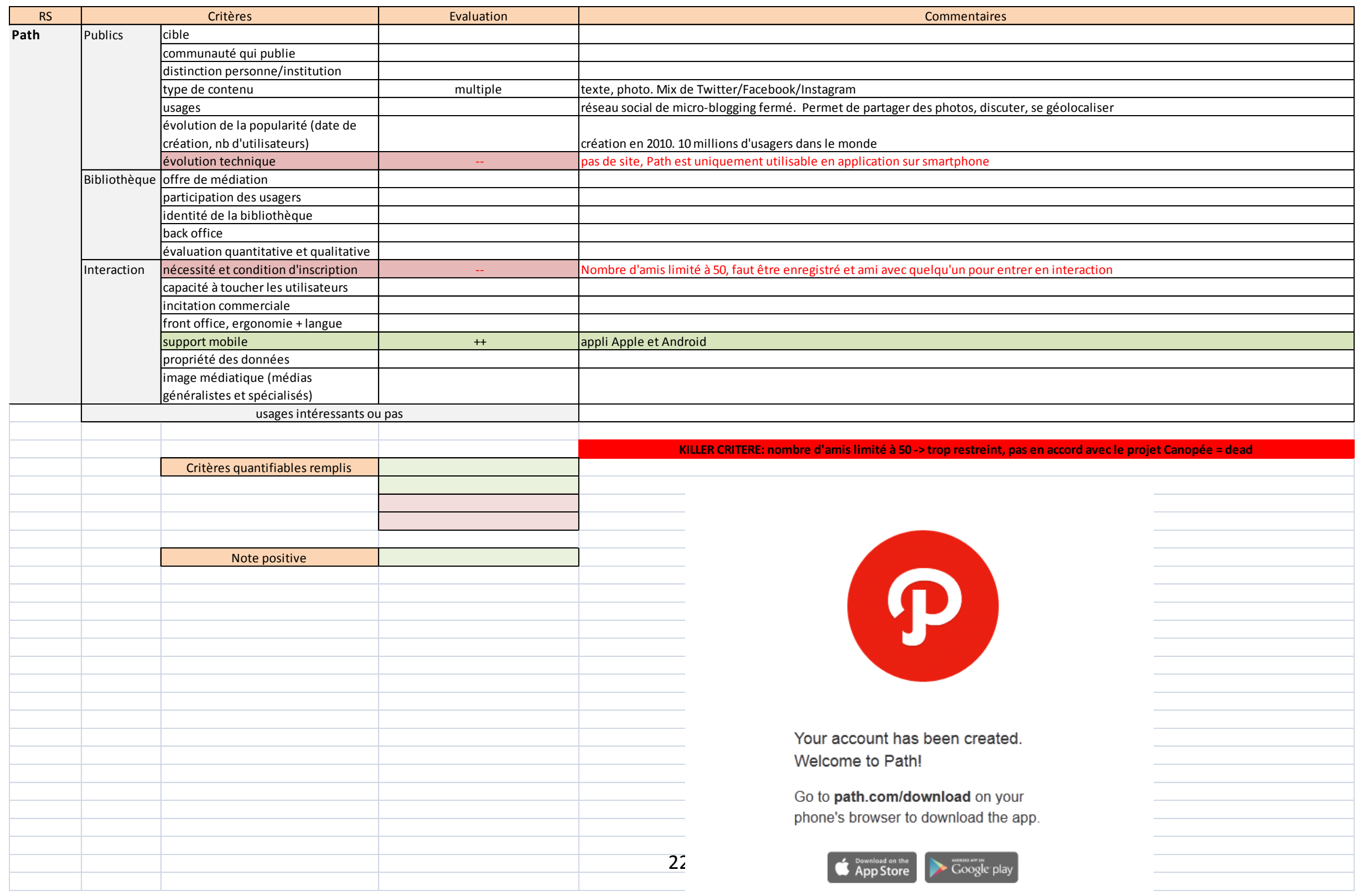




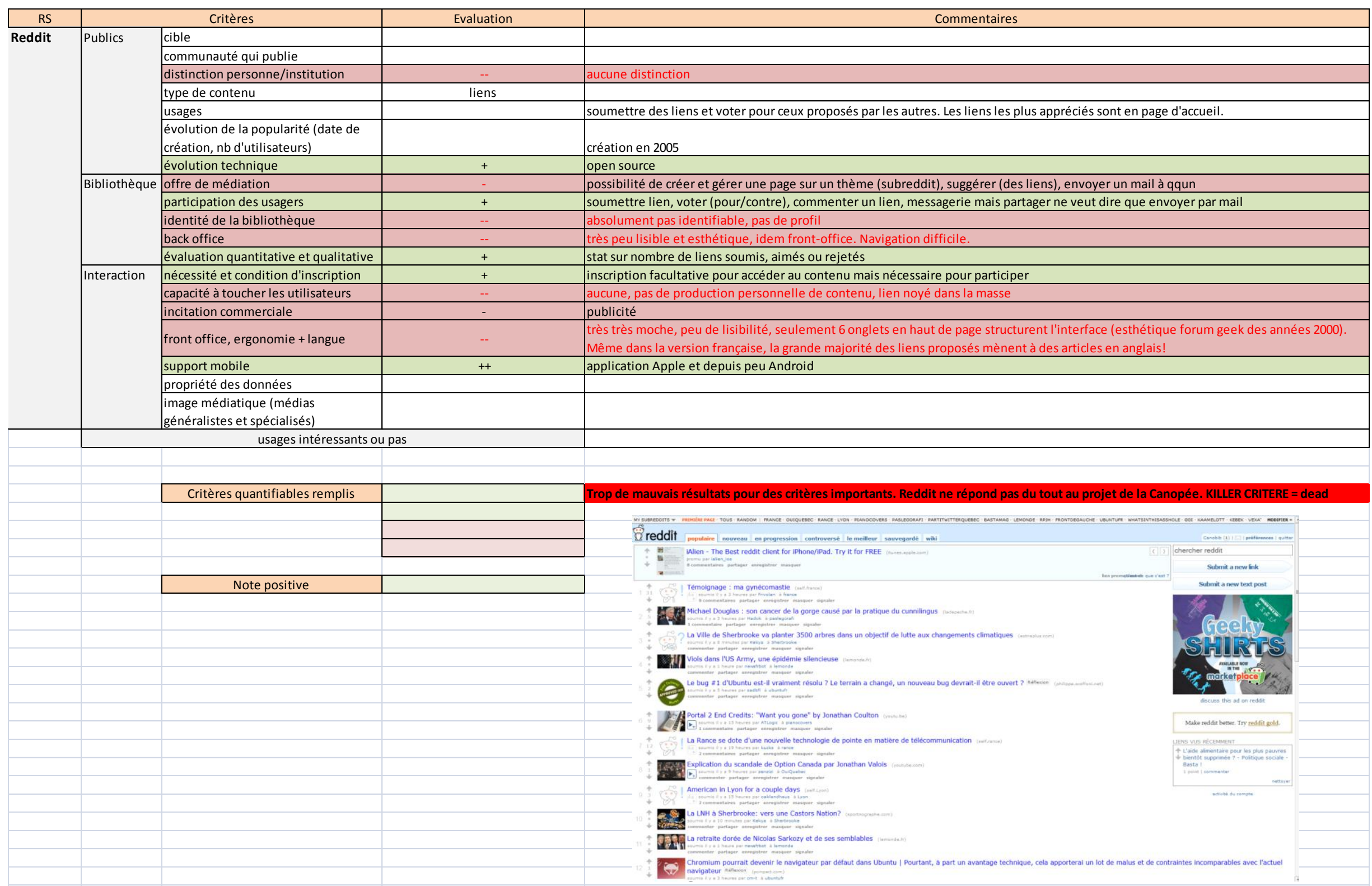




\begin{tabular}{|c|c|c|c|c|}
\hline RS & \multirow{8}{*}{ Publics } & Critères & Evaluation & Commentaires \\
\hline \multirow[t]{19}{*}{ Pheed } & & cible & $\begin{array}{l}\text { jeunes, créateurs, aimant la } \\
\text { nouveauté numérique }\end{array}$ & $\begin{array}{l}\text { 15-24 ans, artistes, avant-gardistes numériques. Volonté de "concurrencer" Twitter et Facebook en permettant de poster dans ces } 2 \\
\text { réseaux depuis sa page d'accueil Pheed. Ressemble à un mix entre Facebook, Twitter, Tumblr et Soundcloud. }\end{array}$ \\
\hline & & communauté qui publie & jeunes, créateurs & $\begin{array}{l}84 \% \text { des utilisateurs ont entre } 15 \text { et 24. Peu de français I'utilisent (cf. pas de version française existante à l'heure actuelle). Artistes, } \\
\text { journalistes, bloggeurs... }\end{array}$ \\
\hline & & distinction personne/institution & ++ & oui, distinction de compte "officiel" \\
\hline & & type de contenu & mutliple & $\begin{array}{l}\text { agrège les fonctions de Facebook, Twitter, Tumblr et Soundcloud. Poster textes ( } 420 \text { caractères), sons, photos, broadcasts, vidéos, sans } \\
\text { limite de temps ni de résolution ou de forme. }\end{array}$ \\
\hline & & usages & $\begin{array}{c}\begin{array}{c}\text { personnel et professionnel, public } \\
\text { et privé }\end{array} \\
\end{array}$ & $\begin{array}{l}\text { Publication de divers médias dans une optique plus qualitative (contenus interactifs et esthétiques privilégiés (photos...)) que } \\
\text { quantitative. Mise en valeur des créations audio, photo et vidéo. Publication pour faire connaître son travail ou ses goûts. }\end{array}$ \\
\hline & & $\begin{array}{l}\text { évolution de la popularité (date de } \\
\text { création, nb d'utilisateurs) }\end{array}$ & + & $\begin{array}{l}\text { création en octobre } 2012 \text { = réseau très jeune. Le } 19 \text { février 2013, Pheed fut la première application téléchargée de l'Apple Store, dépassant } \\
\text { Facebook et Twitter. Plus d'1 million d'utilisateurs en qqs semaines. Semble pourtant pu répandu en France mais vraiment tendance aux } \\
\text { USA. }\end{array}$ \\
\hline & & évolution technique & ++ & réseau encore trop jeune pour avoir des évolutions profondes. Pas de bugs signalés par la communauté d'utilisateurs. \\
\hline & \multirow[t]{5}{*}{ Bibliothèque } & offre de médiation & ++ & $\begin{array}{l}\text { signaler, communiquer, valoriser, discuter. Poster de multiples contenus, Possiblité d'indexer avec les mots clés et hashtag (les plus en } \\
\text { vogue sont en homepage), grande capacité à distribuer les contenus sur d'autres réseaux. }\end{array}$ \\
\hline & & participation des usagers & ++ & $\begin{array}{l}\text { Les « pheeders » peuvent réagir aux contenus en cliquant sur le bouton "I l love this », « i don't love this », « i keep this » (l'équivalent des } \\
\text { favoris sur Twitter) ou encore " remix » (partager). Possibilité d'indexer le contenu posté par les autres. }\end{array}$ \\
\hline & & identité de la bibliothèque & ++ & personnalisable par une photo de profil et de fond, une description. \\
\hline & & back office & ++ & idem front office. Bonne ergonomie. \\
\hline & & évaluation quantitative et qualitative & ++ & indication du nombre de remix, like, don't like, abonnés... pas d'info trouvée sur le reste. \\
\hline & \multirow[t]{7}{*}{ Interaction } & nécessité et condition d'inscription & -- & $\begin{array}{l}\text { il n'est pas possible d'accéder à Pheed depuis son ordinateur, la plateforme existe uniquement comme application. L'inscription est } \\
\text { calquée sur celle de Twitter: il faut choisir des abonnements dès le début. Elle est obligatoire pour accéder à l'appli. }\end{array}$ \\
\hline & & capacité à toucher les utilisateurs & - & $\begin{array}{l}\text { possibilité de limiter l'accès au plus de } 17 \text { ans. Visibilité relative auprès des abonnés car comme Twitter, tout défile... pas plus d'info sur la } \\
\text { capacité à toucher les autres. }\end{array}$ \\
\hline & & incitation commerciale & ++ & $\begin{array}{l}\text { Pheed propose un modèle élaboré de monétisation : chaque membre peut transformer son compte en compte payant pour les autres } \\
\text { utilisateurs, le réseau social ponctionnant une commission, pour du contenu de haute qualité. Intéressant pour les artistes, journalistes, } \\
\text { bloggeurs. L'utilisateur fixe le prix. Pas de pub, car le site vit des commissions et non de la pub comme Facebook. }\end{array}$ \\
\hline & & front office, ergonomie + langue & + & \multirow{2}{*}{$\begin{array}{l}\text { Ia présentation ressemble beaucoup à Twitter, avec sa timeline. Clair et beau. Langue anglaise pour l'instant! } \\
\text { appli Android et Apple }\end{array}$} \\
\hline & & support mobile & ++ & \\
\hline & & propriété des données & ++ & $\begin{array}{l}\text { appli Androld et Apple } \\
\text { Pheed n'a aucun droit sur les contenu et propose même aux utilisateurs de mettre un copyright }\end{array}$ \\
\hline & & $\begin{array}{l}\text { image médiatique (médias } \\
\text { généralistes et spécialisés) }\end{array}$ & ++ & $\begin{array}{l}\text { très bonne image, bonne surprise. Points forts: fonctionnalités très étendues, protection des contenus et proriétés des données (le gros } \\
\text { point faible de Facebook notamment), côté branché qui va de paire avec l'usage encore peu massif de l'application / points faibles: quel } \\
\text { avenir en France? }\end{array}$ \\
\hline & \multicolumn{3}{|c|}{ usages intéressants ou pas } & réseau à surveiller dans son évolution. Permet de diffuser de nombreux types de contenus, avec une image qualitative forte. \\
\hline & & & & pheed \\
\hline & & Critères quantifiables remplis & $11++/ 30$ & \\
\hline & & & $2+/ 30$ & A new way to express \\
\hline & & & $1-/ 30$ & text, photo, video, audio, broadcast \\
\hline & & & $1--/ 30$ & 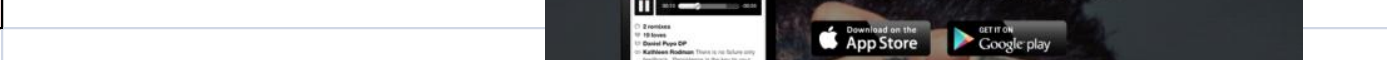 \\
\hline & & Note positive & $21 / 30$ & $\mathrm{Da}$ \\
\hline & & & & $a^{2}=-$ \\
\hline & & & & 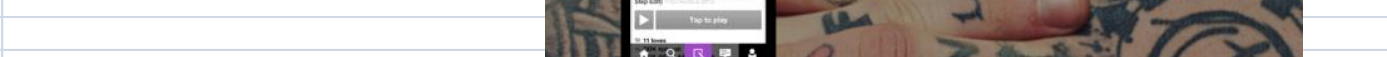 \\
\hline & & & & \\
\hline & & & & 2) \\
\hline & & & & \\
\hline
\end{tabular}




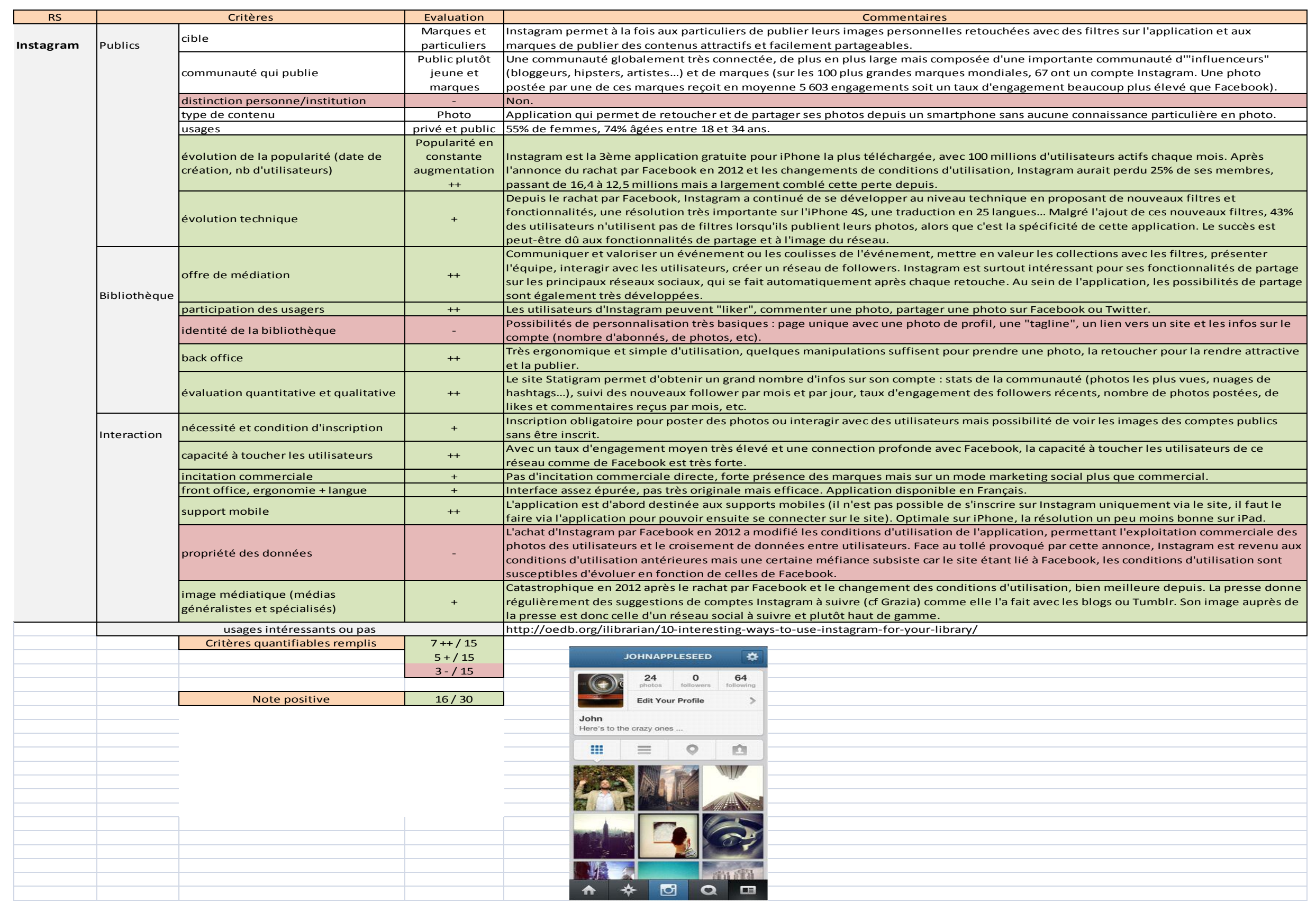




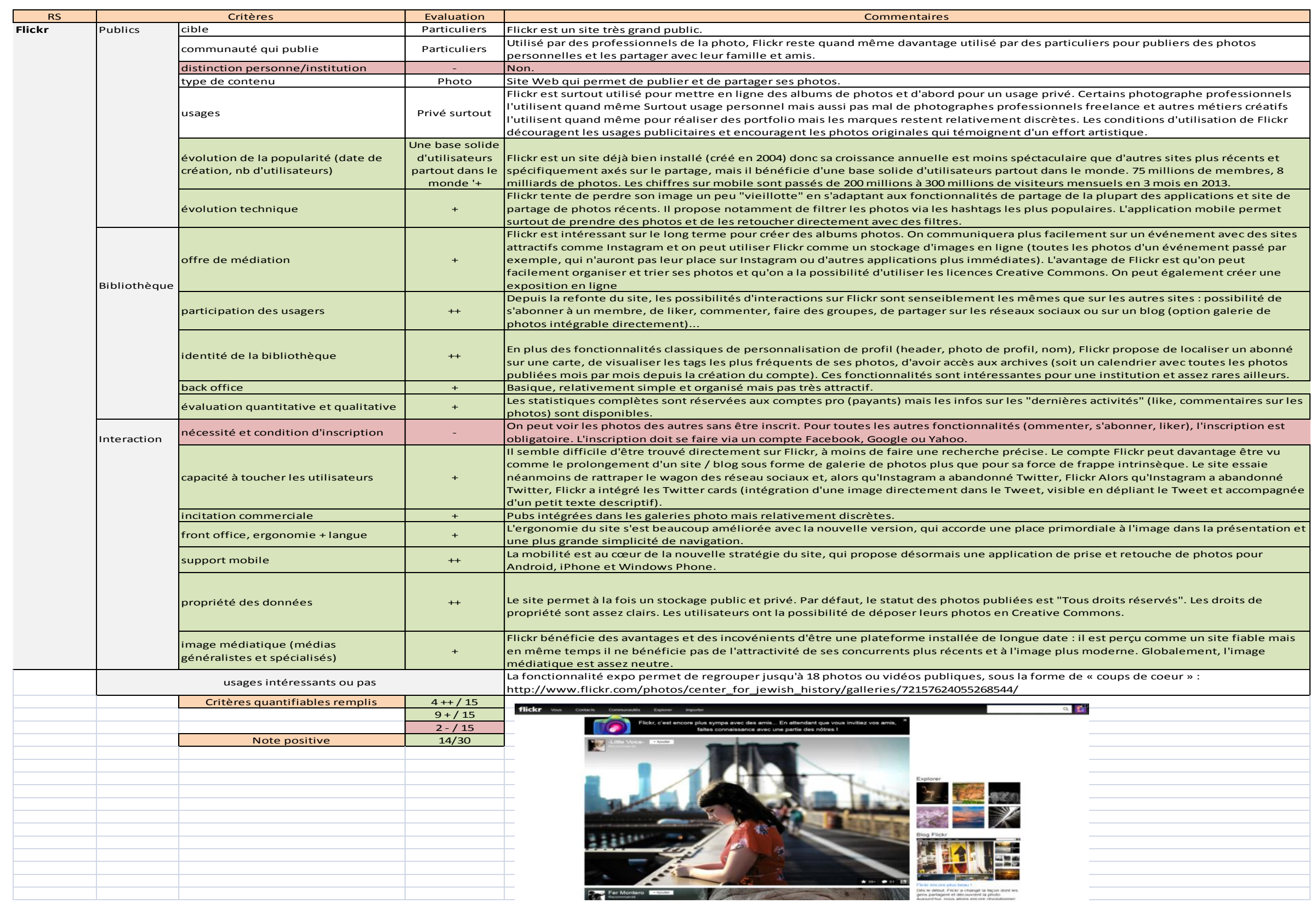




\begin{tabular}{|c|c|c|c|}
\hline \multirow{3}{*}{\begin{tabular}{|c|} 
RS \\
Youtube
\end{tabular}} & \multicolumn{2}{|r|}{ Critères } & \multirow{3}{*}{\begin{tabular}{|c|} 
Evaluation \\
$\begin{array}{l}\text { Particuliers et } \\
\text { professionnel }\end{array}$ \\
Tout le mond
\end{tabular}} \\
\hline & \multirow{7}{*}{ Publics } & cible & \\
\hline & & communauté qui publie & \\
\hline & & distinction personne/institution & - \\
\hline & & type de contenu & Vidéo \\
\hline & & usages & Perso et pro \\
\hline & & $\begin{array}{l}\text { évolution de la popularité (date de } \\
\text { création, nb d'utilisateurs) }\end{array}$ & ++ \\
\hline & & évolution technique & ++ \\
\hline & \multirow[t]{5}{*}{ Bibliothèque } & offre de médiation & + \\
\hline & & participation des usagers & + \\
\hline & & identité de la bibliothèque & + \\
\hline & & back office & + \\
\hline & & évaluation quantitative et qualitative & + \\
\hline & \multirow{7}{*}{ Interaction } & nécessité et condition d'inscription & + \\
\hline & & capacité à toucher les utilisateurs & ++ \\
\hline & & incitation commerciale & -- \\
\hline & & front office, ergonomie + langue & + \\
\hline & & support mobile & ++ \\
\hline & & propriété des données & - \\
\hline & & $\begin{array}{l}\text { image médiatique (médias } \\
\text { généralistes et spécialisés) }\end{array}$ & + \\
\hline & \multicolumn{3}{|c|}{ usages intéressants ou pas } \\
\hline & & Critères quantifiables remplis & $\frac{6++/ 15}{7+/ 15}$ \\
\hline & & & $1-/ 15$ \\
\hline & & & $1--/ 15$ \\
\hline & & Note positive & $14 / 30$ \\
\hline & & & \\
\hline & & & \\
\hline & & & \\
\hline & & & \\
\hline & & & \\
\hline & & & \\
\hline & & & \\
\hline & & & \\
\hline & & & \\
\hline
\end{tabular}

Tout le monde

Particuliers, marques, institutions, groupes de musique, etc

as de réelle distinction entre personne privée et institution mais possibilité de créer des "chaînes" (utilisées par les bloggeurs, les marques, les institutions, etc) classées par thématique. Les chaînes Youtube sont gratuites et peuvent être personnalisée et partagées acilement. Des études (dernière étude baromètre Hadopi) ont montré que les contenus amateurs ont tendance à s'effacer peu à peu au profit des chaines qui offrent globalement des contenus a plus forte valeur ajoutée.

The extraits de films, deemissions de télé, clips, etc... Contenus très variés. intéressante pour les marques et les institutions, avec quand même encore une grande part de contenus amateurs. 12 millitine céateurs énèrent des revenus à pantir de leur avivité sur Youtube créateurs génèrent des revenus à partir de leur activité sur Youtube.

列 3 des pages vues mondiales, avec 800 millions de isiteurs par mois et 1 milliard d'abonnés à des chaînes Youtube en 2010.

lateforme relativement stable mais évolutions techniques régulières dans le sens d'une plus grande ergonomie et de nouvelles Oréer dun lités de partage (nouvelle interface en 2013)

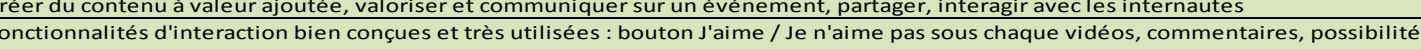
de voir ce que regardent ses amis Facebook sans quitter Youtube, boutons de partage classiques... Les commentaires sont particulièrement utilisés sur Youtube, avec plus de $50 \%$ de l'ensemble des vidéos commentées par la communauté. Les vidéos qu'on a imé" sont sauvegardées et restent accessibles depuis son compte.

er personnalisable, surtout les chaînes (header, $A$ propos, photo de profil, vidéo à la Une, galerie de vidéos, Playlist...) mais la

Beaucoup d'évolutions récentes pour plus de simplicité et un plus grand confort dans l'administration (la présence de Google derrière Youtube fait que la plateforme bénéficie forcément de moyens importants pour rendre l'utilisation la plus intuitive possible). Néanmoins, le back office reste un peu plus complexe et fouilli que d'autres plateformes qui misent vraiment sur la sobriété (Vimeo). Youtube propose un nombre de services très important donc la gestion de ces outils est peut-être un peu moins simple.

intégration de Google analytics pour les stats, l'un des outils les plus utiles, intuitifs et complets en matières de statistiques Web (stats sur 'ensemble de la chaine ou par vidéo, nombre de likes, de favoris, de partage...)

La plupart des vidéos du site ou les chaines You Tube peuvent être visualisées et partagées par tous les internautes, tandis que seules les ersonnes inscrites peuvent envoyer des vidéos de façon illimitée. II faut être inscrit pour pouvoir commenter et noter des vidéos.

permet d'afficher des vidéos similaires ce qui rend la navigation fluide et multiplie les chances de toucher un publicciblé. e format publicitaire "instreamil, intégré quirectement au début de la vidéo, est très intrusif. Le modèle économique de la plateforme est basé sur la publicité donc les incitations commerciales sont forcément fortes et dépendent de la popularité du contenu. a nouvelle version de 2013 a complètement modifié l'interface et l'expérience de navigation, au profit d'une plus grande sobriété. Le résultat est beaucoup plus ergonomique. La plateforme est disponible en 61 langues. qui cherche encore à étendre son influence sur ce support.

d'utilisateur conserve les droits de proprieté intelectuelle sur son contenu mais est tenu de céder des droits limités à Youtube : droit d'utiliser, de reproduire, de distribuer, de réaliser des œuvres dérivées de tout ou partie du contenu

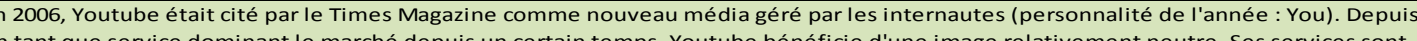
tilisés par la plupart des grands médias pour diffuser des vidéos. http://www.youtube.com/user/LibraryOfCongress

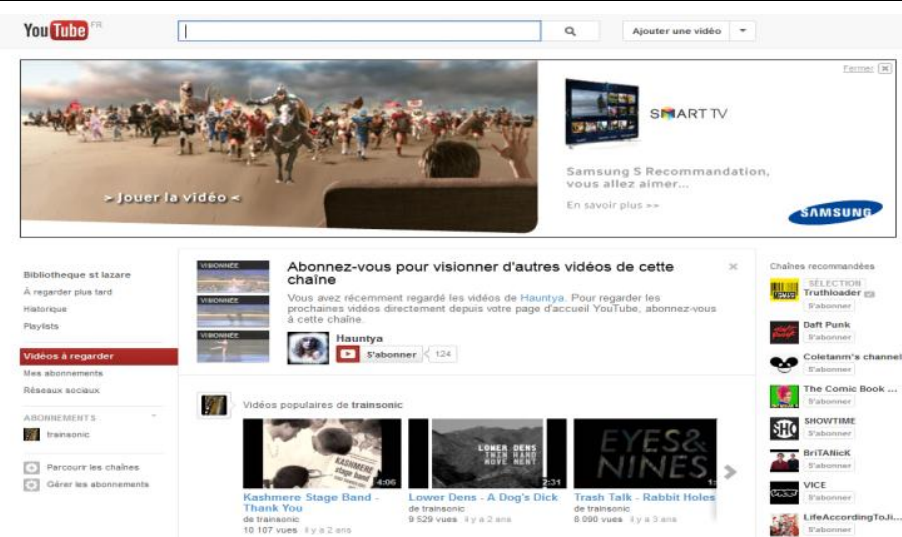




\begin{tabular}{|c|c|c|c|c|}
\hline RS & & Critères & Evaluation & Commentaires \\
\hline \multirow[t]{19}{*}{ Dailymotion $\mathrm{P}$} & \multirow[t]{7}{*}{ Publics } & cible & $\begin{array}{l}\text { Particuliers et } \\
\text { professionnels }\end{array}$ & Tout le monde \\
\hline & & communauté qui publie & Tout le monde & $\begin{array}{l}\text { Particuliers, marques, institutions, groupes de musique, etc. La plateforme cherche à attirer les utilisateurs créatifs (acteur, musiciens, } \\
\text { réalisateurs de clips, de court métrages, de webséries Etc...), soit les "motionmakers", qui ont la possibilité d'envoyer leurs vidéos comme } \\
\text { Creative Content (contenu créatif). Cela veut dire qu'ils mettent leurs vidéos à la disposition de l'équipe éditoriale de Dailymotion, qui } \\
\text { peut ensuite décider de mettre en avant la vidéo sur la page d'accueil du site (vidéo star). }\end{array}$ \\
\hline & & distinction personne/institution & - & (a) \\
\hline & & type de contenu & Vidéo & $\begin{array}{l}\text { Vidéos amateures en majorité mais aussi extraits de films, d'émissions de télé, clips, beaucoup de dessins animés et de séries (extraits de } \\
\text { séries TV très populaires, deux fois moins de clips musicaux que sur Youtube) }\end{array}$ \\
\hline & & usages & $\begin{array}{l}\text { Particuliers et } \\
\text { professionnels }\end{array}$ & Youtube est plus populaire que Dailymotion auprès des entreprises. \\
\hline & & $\begin{array}{l}\text { évolution de la popularité (date de } \\
\text { création, nb d'utilisateurs) }\end{array}$ & ++ & $\begin{array}{l}\text { 31ème site le plus visité dans le monde avec } 112 \text { millions de visiteurs uniques, } 2,5 \text { milliards de vidéos vues par mois et une croissance de } \\
100 \% \text { en 2012, par rapport à 2011. Premier site Français en termes d'audience. }\end{array}$ \\
\hline & & évolution technique & ++ & $\begin{array}{l}\text { Plateforme relativement stable qui garde ses repères pour les utilisateurs mais proposent très souvent de nouvelles fonctionnalités. Son } \\
\text { lecteur intégré est l'un des plus performant, le Mass Uploader permet de télécharger un grand nombre de vidéos en même temps... }\end{array}$ \\
\hline & \multirow[t]{5}{*}{ Bibliothèque } & offre de médiation & + & Créer du contenu à valeur ajoutée, valoriser et communiquer sur un événement, partager, interagir avec les internautes \\
\hline & & participation des usagers & ++ & $\begin{array}{l}\text { Les vidéos sont accompagnées de boutons de partage classiques Twitter et Facebook mais les autres réseaux sont cachés sous forme d'un } \\
\text { menu déroulant quasi invisible, possibilité de commenter les vidéos mais fonctions de partage relativement moins développées et moins } \\
\text { mises en valeur que sur d'autres plateformes. }\end{array}$ \\
\hline & & identité de la bibliothèque & ++ & $\begin{array}{l}\text { Les possibilités de customisation de l'interface intéressantes (background, header, image de profil, longue description, relié à tous les } \\
\text { comptes Facebook, Twitter, avec boutons "suivre"). La plupart des chaînes TV et des fournisseurs de contenus ont leur chaîne. Il est assez } \\
\text { facile de mettre en valeur l'identité de la bibliothèque sur cette plateforme. }\end{array}$ \\
\hline & & back office & + & $\begin{array}{l}\text { Deux clics suffisent pour accéder au menu et commencer à télécharger, ce qui n'est pas le cas pour les services concurrents. Sur la page de } \\
\text { téléchargement, on accède à une liste d'options classiques pour classer la vidéo, la taguer, écrire une courte description, etc }\end{array}$ \\
\hline & & évaluation quantitative et qualitative & + & $\begin{array}{l}\begin{array}{l}\text { Statistiques en temps réel du nombre de vues d'une vidéo, des moments où elle a été le plus regardée, de la note moyenne des } \\
\text { internautes, du nombre de commentaires, etc }\end{array}\end{array}$ \\
\hline & \multirow[t]{7}{*}{ Interaction } & nécessité et condition d'inscription & + & On peut voir et partager les vidéos sans être inscrits mais les fonctionnalités de partage et de commentaires nécessitent l'inscription. \\
\hline & & capacité à toucher les utilisateurs & ++ & $\begin{array}{l}\text { Forte capacité à toucher les utilisateurs notamment avec l'activity feed, fil d'information présent sur toutes les pages qui affiche en temps } \\
\text { réel les activités des comptes que l'on suit (vidéos postées mais aussi commentaires, like, etc). Les utilisateurs inscrits avec Facebook } \\
\text { connect voient également dans ce fil les activités de leurs amis Facebook. }\end{array}$ \\
\hline & & incitation commerciale & -- & $\begin{array}{l}\text { Le format publicitaire "instream", intégré directement au début de la vidéo, est très intrusif. Le modèle économique de la plateforme est } \\
\text { basé sur la publicité donc les incitations commerciales sont forcément fortes et dépendent de la popularité du contenu. }\end{array}$ \\
\hline & & front office, ergonomie + langue & + & $\begin{array}{l}\text { Page d'accueil assez peu accueillante, avec background publicitaire et défilement sans fin de vidéos sous différents formats. La navigation } \\
\text { sur le site est fluide. Le site est disponible en } 16 \text { langues. }\end{array}$ \\
\hline & & support mobile & ++ & Application mobile pour iPhone, iPad, Android et Windows Phone. \\
\hline & & propriété des données & + & $\begin{array}{l}\text { Dailymotion déclare expressément dans ses conditions d'utilisation n'avoir aucun droit sur le contenu de ses membres (vidéos, avatar, } \\
\text { commentaires...). La plateforme se réserve quand même le droit de "reproduire/représenter" le contenu }\end{array}$ \\
\hline & & $\begin{array}{l}\text { image médiatique (médias } \\
\text { généralistes et spécialisés) }\end{array}$ & + & $\begin{array}{l}\text { En France, l'image de Dailyotion est plutôt positive : un site "made in France" qui arrive à concurrencer le géant américain Youtube. Son } \\
\text { image dans les médias n'est pas particulièrement innovante en termes de contenus ou de fonctionnalités. Dailymotion a été récemment } \\
\text { sur le devant de la scène avec la volonté de rachat par Yahoo et le refus de Montebourg de céder à une entreprise Américaine le capital } \\
\text { d'une entreprise française. }\end{array}$ \\
\hline & \multicolumn{3}{|c|}{ usages intéressants ou pas } & \multirow{2}{*}{$\begin{array}{l}\text { http://www.dailymotion.com/Bpi_Centre_Pompidouttvideo=xzbcfz } \\
\text { Dailymofion }\end{array}$} \\
\hline & & Critères quantifiables remplis & $6++/ 15$ & \\
\hline & & & \begin{tabular}{|l|l|l|l|l}
$7+/ 15$ \\
$1-/ 15$
\end{tabular} & 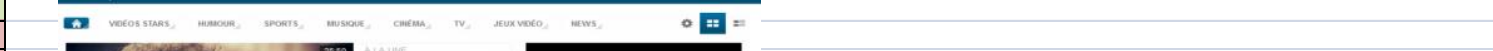 \\
\hline & & & $1--/ 15$ & \\
\hline & & Note positive & $16 / 30$ & \\
\hline & & & & \\
\hline & & & & \\
\hline & & & & $E$ \\
\hline & & & & tome: \\
\hline & & & & - \\
\hline & & & & 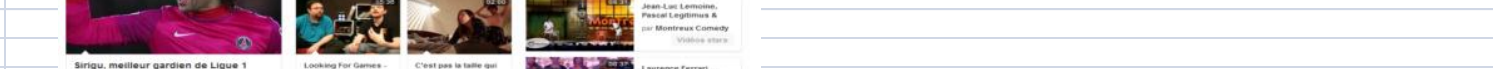 \\
\hline & & & & 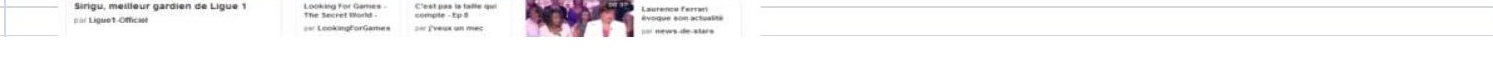 \\
\hline
\end{tabular}




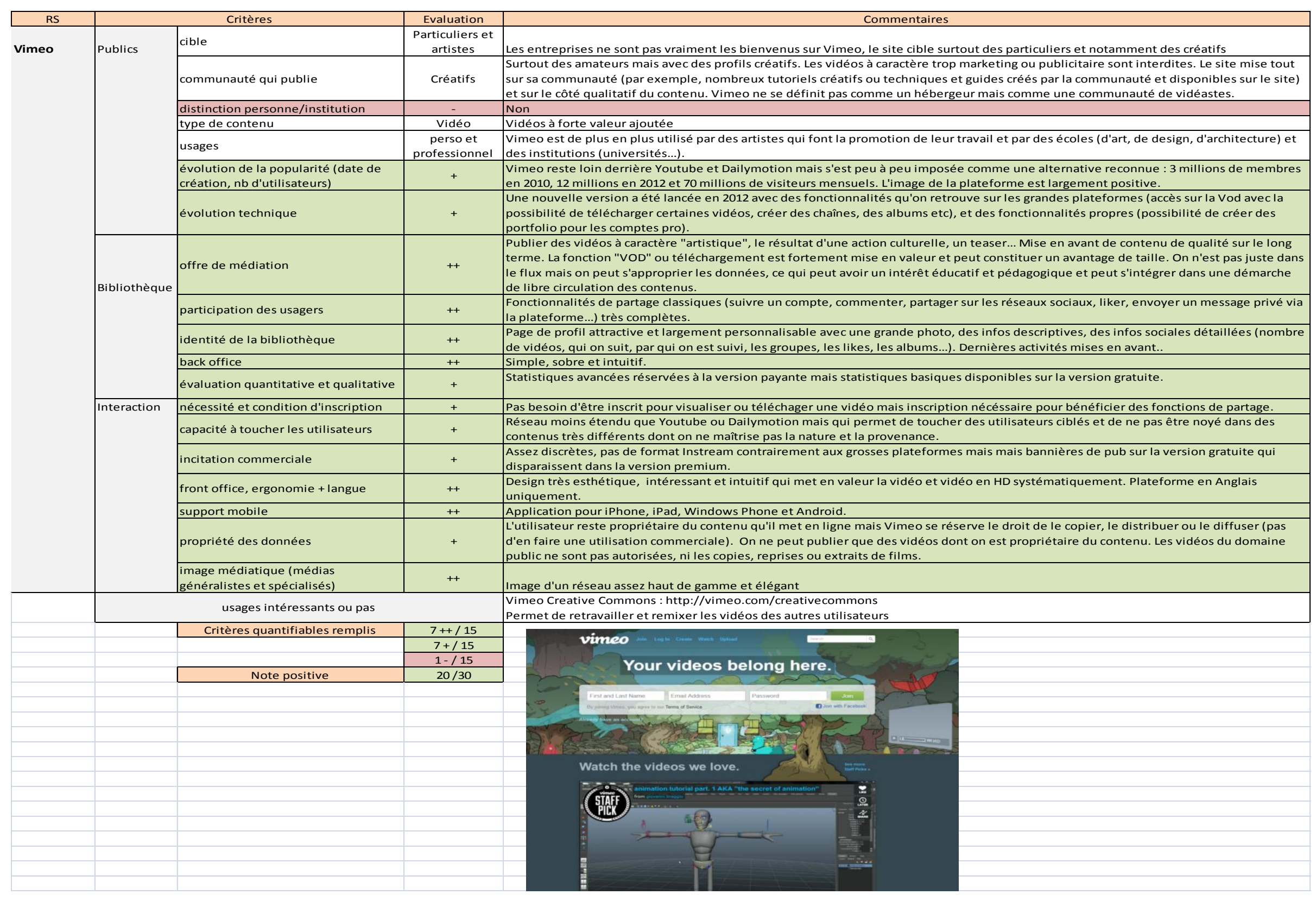




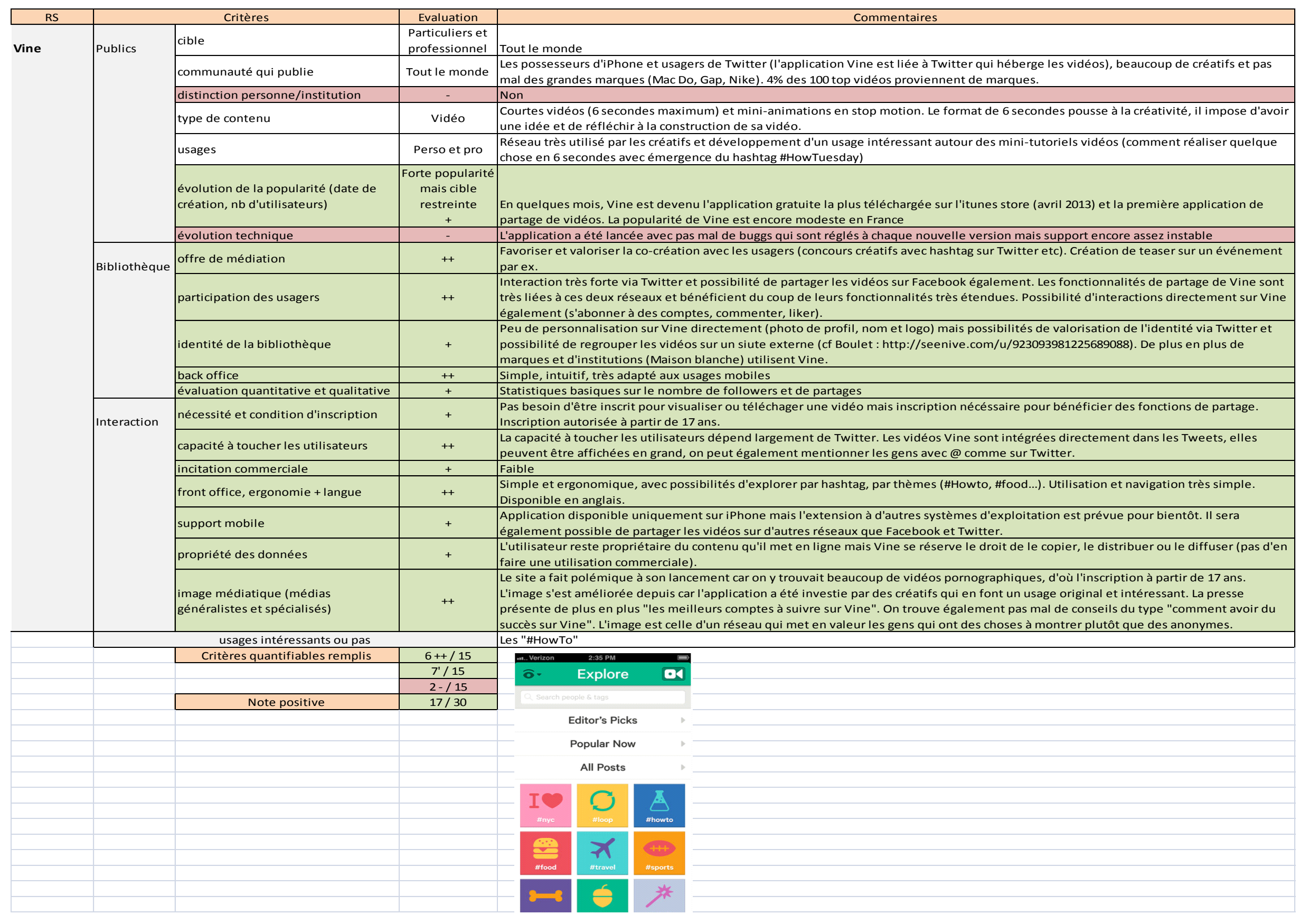




\begin{tabular}{|c|c|c|c|c|}
\hline \multirow{3}{*}{\begin{tabular}{l}
\multicolumn{1}{c}{ RS } \\
Myspace \\
version \\
classique
\end{tabular}} & \multicolumn{2}{|r|}{ Critères } & \multicolumn{2}{|r|}{ Commentaires } \\
\hline & \multirow[t]{7}{*}{ Publics } & cible & particulier et musicien & on choisit l'un ou l'autre à l'inscription \\
\hline & & communauté qui publie & groupe de musique & \\
\hline & & distinction personne/institution & - & pas de distinction institutionnelle possible (il est obligatoire de dire si l'on est fille ou garçon notamment) \\
\hline & & type de contenu & multiple & musique, photos, texte, vidéo \\
\hline & & usages & semi-professionnel & $\begin{array}{l}\text { les petits groupes et artistes amateurs ou tout juste pro utilisaient Myspace comme un site perso (mais ils préfèrent aujourd'hui d'autres } \\
\text { plateforme). Le site leur permet de diffuser leur musique, se faire connaître et faire leur promo. }\end{array}$ \\
\hline & & $\begin{array}{l}\text { évolution de la popularité (date de } \\
\text { création, nb d'utilisateurs) }\end{array}$ & - & $\begin{array}{l}\text { créé en 2003, énorme succès du site au début. Pas de chiffres récents. Dans I'opinion commune, Myspace est devenu as been, dépassé par } \\
\text { les Facebook et Cie. Le site semble abandonné par les artistes connus (pas de connexion depuis des années) }\end{array}$ \\
\hline & & évolution technique & + & Une nouvelle version Beta de Myspace est mise en service en janvier 2013 qui se veut très design et super à la mode. \\
\hline & \multirow[t]{5}{*}{ Bibliothèque } & offre de médiation & ++ & $\begin{array}{l}\text { conseiller (faire une playlist), suggérer (partager un morceau), valoriser (un événement musical en photo), communiquer (calendrier } \\
\text { d'événement à venir), écrire (une fonction blog) }\end{array}$ \\
\hline & & participation des usagers & - & possibilité de partager, envoyer un mail privé, mettre des commentaires: restreint. \\
\hline & & identité de la bibliothèque & ++ & page personnalisable, couleur, photo de profil, description \\
\hline & & back office & ++ & $\begin{array}{l}\text { beaucoup de possibilités de personnalisation du profil utilisateur (agencement des encarts, style média social, style musique, style } \\
\text { vidéo...). Interface assez simple }\end{array}$ \\
\hline & & évaluation quantitative et qualitative & ++ & statistiques gratuites pour le nombre de vues de la page, nombre d'écoute d'un morceau \\
\hline & \multirow[t]{7}{*}{ Interaction } & nécessité et condition d'inscription & + & pas nécessaire de s'inscrire pour avoir accès aux pages. II faut se connecter pour laisser un commentaire. \\
\hline & & capacité à toucher les utilisateurs & -- & pour recevoir les infos, les utilisateurs doivent demander à être ami avec le profil de qqun: envoie une invitation qu'il faut accepter \\
\hline & & incitation commerciale & -- & beaucoup de pub, deux grandes banières animées de chaque côté de la page et en bas \\
\hline & & front office, ergonomie + langue & -. & $\begin{array}{l}\text { deux plateformes proposées: la classique (en français) ou la nouvelle (en anglais). Le lecteur de musique s'ouvre dans une autre fenêtre } \\
\text { (ne marche pas sur les ordi de la Ville car flash player pas mis à jour). Plusieurs onglets: musique, vidéo, jeux, photos, amis. La nouvelle } \\
\text { version est incompréhensible, très très dur de s'y repérer, change totalement de la version classique. Pas de retours officiels sur la mise en } \\
\text { service unique de la nouvelle version. }\end{array}$ \\
\hline & & support mobile & ++ & application Apple et Android \\
\hline & & propriété des données & -. & aucune info trouvée, même sur le site lui-même \\
\hline & & $\begin{array}{l}\text { image médiatique (médias } \\
\text { généralistes et spécialisés) }\end{array}$ & - & $\begin{array}{l}\text { médias spécialisés ont fait un accueil chaleureux au design de la nouvelle version. Les médias se demandent si Myspace va réussir à } \\
\text { remonter la pente de la popularité. Avant cette nouvelle version, Myspace était absent des médias. }\end{array}$ \\
\hline & \multicolumn{3}{|c|}{ usages intéressants ou pas } & $\begin{array}{l}\text { Les bibliothèques sont absentes de Myspace. La dernière publication de la british library (vraiment active sur les réseaux sociaux) date d'il } \\
\text { y a } 5 \text { ans! Les artistes aussi ont déserté la plateforme... Tous les profils visités n'affichent AUCUNE activité récente. }\end{array}$ \\
\hline
\end{tabular}

Critères quantifiables remplis

\begin{tabular}{|c|}
\hline $5++$ \\
\hline $2+$ \\
\hline $2-$ \\
\hline $6--$ \\
\hline
\end{tabular}

Note positive

$-1$

\section{myspace Accuell Profll Trucs persor 10 a}

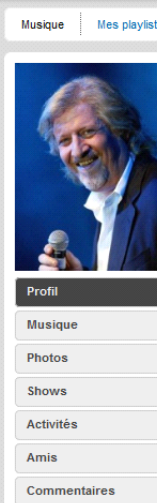

Patrick Sébastien

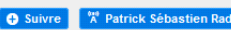

Musique

Ala une Morceaux Playilists

Infos générales

Geere: Chanson fra
Lieu Limousin, $F R$

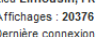

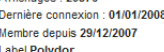

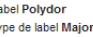

$+\mathrm{T}$
Nombre totat de lectures : 2790 / Hombre de lectures asjourchnui: 1 Tour

Activités

Tout Musique Videos photos sujets +.
붕하

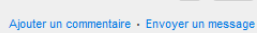

numericable

assez au

ès haut

ébit!

Internet
jusqu'à
jug

200 méga

Member Since:
oeceneref 2 2.2077
Influences: 


\begin{tabular}{|c|c|c|c|c|}
\hline RS & & Critères & Evaluation & Commentaires \\
\hline \multirow[t]{19}{*}{ Soundcloud } & \multirow[t]{7}{*}{ Publics } & cible & $\begin{array}{l}\text { musiciens et professionnels de la } \\
\text { musique }\end{array}$ & musiciens amateurs et professionnels, professionnels de l'édition musicale \\
\hline & & communauté qui publie & musiciens, particuliers & musiciens qui souhaitent se faire découvrir et/ou partager leur création, particuliers qui souhaitent écouter de la musique en streaming \\
\hline & & distinction personne/institution & + & pas de page distinguée personne / institution mais possibilité de mettre une photo et de se décrire, profil succinct \\
\hline & & type de contenu & audio & $\begin{array}{l}\text { musique mais aussi émission de radio (sport, actualité, littérature...), comédie, livre audio et même des cours (catégorie learning, cours de } \\
\text { I'université d'Havard par exemple) }\end{array}$ \\
\hline & & usages & $\begin{array}{l}\text { privé (pour le loisirs), } \\
\text { professionnel }\end{array}$ & $\begin{array}{l}\text { permet de publier de la musique ailleurs que sur sa page, d'intégrer des fichiers audio dans une page web (notamment Facebook et } \\
\text { Twitter). Les musiciens s'en servent pour s'échanger des séquences musicales, collaborer et faire leur promotion. Le grand public vient y } \\
\text { découvrir des nouveaux groupes (écoute en streaming et téléchargement de pistes audio), se créer des playlists, participer à des groupes } \\
\text { (échange de musique). Le site propose un espace de stockage "cloud", 2h gratuites. }\end{array}$ \\
\hline & & $\begin{array}{l}\text { évolution de la popularité (date de } \\
\text { création, nb d'utilisateurs) }\end{array}$ & ++ & Iancé en 2007, 10 millions d'utilisateurs enregistrés (janvier 2012), 188 millions d'utilisateurs (décembre 2012) \\
\hline & & évolution technique & ++ & $\begin{array}{l}\text { widget à insérer dans une page web, développement d'applications open source, fichiers audio peuvent mis en ligne et téléchargés } \\
\text { depuis un smartphone, procédé API (application programming interface) [à creuser: une appli permet d'organiser un concours de remix, } \\
\text { en mettant en place un système de vote et de partage des remix proposés par vos auditeurs (remix contest Zero 7)] }\end{array}$ \\
\hline & \multirow[t]{5}{*}{ Bibliothèque } & offre de médiation & ++ & $\begin{array}{l}\text { valoriser, signaler, suggérer, échanger par mail. création de playlist, créer un groupe, organiser les contacts par groupe et définir des listes } \\
\text { d'accès précises pour chacun des fichiers audio. II ne semble pas possible de décrire un morceau autrement qu'en le commentant mais } \\
\text { tout de même une possibilitié d'indexation avec les tag. }\end{array}$ \\
\hline & & participation des usagers & ++ & $\begin{array}{l}\text { liker, ajouter à une playlist, partager sur d'autres réseaux sociaux, suivre/être suivi, envoyer un message privé, possibilité d'ajouter un } \\
\text { commentaire à un endroit précis de la séquence audio, d'envoyer des musiques à d'autres directement sur leur compte Soundcloud via } \\
\text { Dropbox, s'abonner à un groupe }\end{array}$ \\
\hline & & identité de la bibliothèque & ++ & $\begin{array}{l}\text { photo, description, faire apparaître de façon visible les liens vers la page de son site, et les pages sur différents réseaux sociaux (Facebook, } \\
\text { Google +, Twitter, Tumblr, You Tube...) }\end{array}$ \\
\hline & & back office & ++ & clair et simple d'utilisation \\
\hline & & évaluation quantitative et qualitative & + & suivi statistique par titre: payant mais gratuit pour nombre d'écoute, de commentaires, de favoris, de téléchargements... \\
\hline & \multirow[t]{7}{*}{ Interaction } & nécessité et condition d'inscription & ++ & on peut diffuser de la musique auprès de gens qui ne sont pas inscrits, possibilité de se connecter via Facebook \\
\hline & & capacité à toucher les utilisateurs & + & on peut reposter une piste audio sur notre mur ou celui de nos followers \\
\hline & & incitation commerciale & ++ & services payants (fonctions avancées) mais la version gratuite offre suffisamment de fonctionnalités. Modèle "freemiuim": pas de pub \\
\hline & & front office, ergonomie + langue & + & $\begin{array}{l}\text { Interface simple et claire, recherche de musique par genre musical en page d'accueil ou barre de recherche, proposition avec jaquette. } \\
\text { Anglais }\end{array}$ \\
\hline & & support mobile & ++ & appli pour iPhone, iPad et Android (tablettes et smartphones) \\
\hline & & propriété des données & + & $\begin{array}{l}\text { pas d'exploitation des données privées des utilisateurs, Creative Commons MAIS incertitude sur l'avenir juridique du site car il héberge } \\
\text { des contenus sans en détenir les droits d'auteur + cf article Calimaq http://scinfolex.wordpress.com/2013/04/18/filtrage-soundcloud-fait- } \\
\text { sa-police-du-copyright }\end{array}$ \\
\hline & & $\begin{array}{l}\text { image médiatique (médias } \\
\text { généralistes et spécialisés) }\end{array}$ & ++ & \multirow[b]{2}{*}{$\begin{array}{l}\text { très bonne image professionnelle } \\
\text { animation de groupes selon les genres musicaux, faire découvrir de nouvelles créations au public. Si à terme le site se "francise" (et donc } \\
\text { des contenu audio autre que musique en Français), pourquoi ne pas constituer une sorte de sélection de podcast sur différentes } \\
\text { thématiques, en fonction de l'action culturelle notamment. Très peu de bib présentes aujourd'hui. }\end{array}$} \\
\hline & \multicolumn{3}{|c|}{ usages intéressants ou pas } & \\
\hline & & Critères quantifiables remplis & $10++$ & willili stream" Emplore \\
\hline & & & $5+$ & \\
\hline & & & $0-$ & 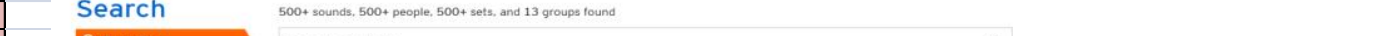 \\
\hline & & & $0--$ & johnny halliday \\
\hline & & Note positive & $25 / 30$ & Sets \\
\hline & & & & is Groups \\
\hline & & & & 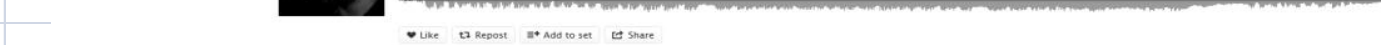 \\
\hline & & & & \\
\hline & & & & Johnny Hallitay - Je Te Promet ( Cover) \\
\hline & & & & \\
\hline & & & & 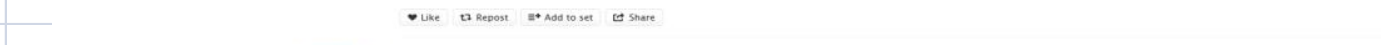 \\
\hline & & & & O $_{\text {Imitation den de Johnny Halliday }}^{\text {Firat }}$ \\
\hline & & & & \\
\hline
\end{tabular}




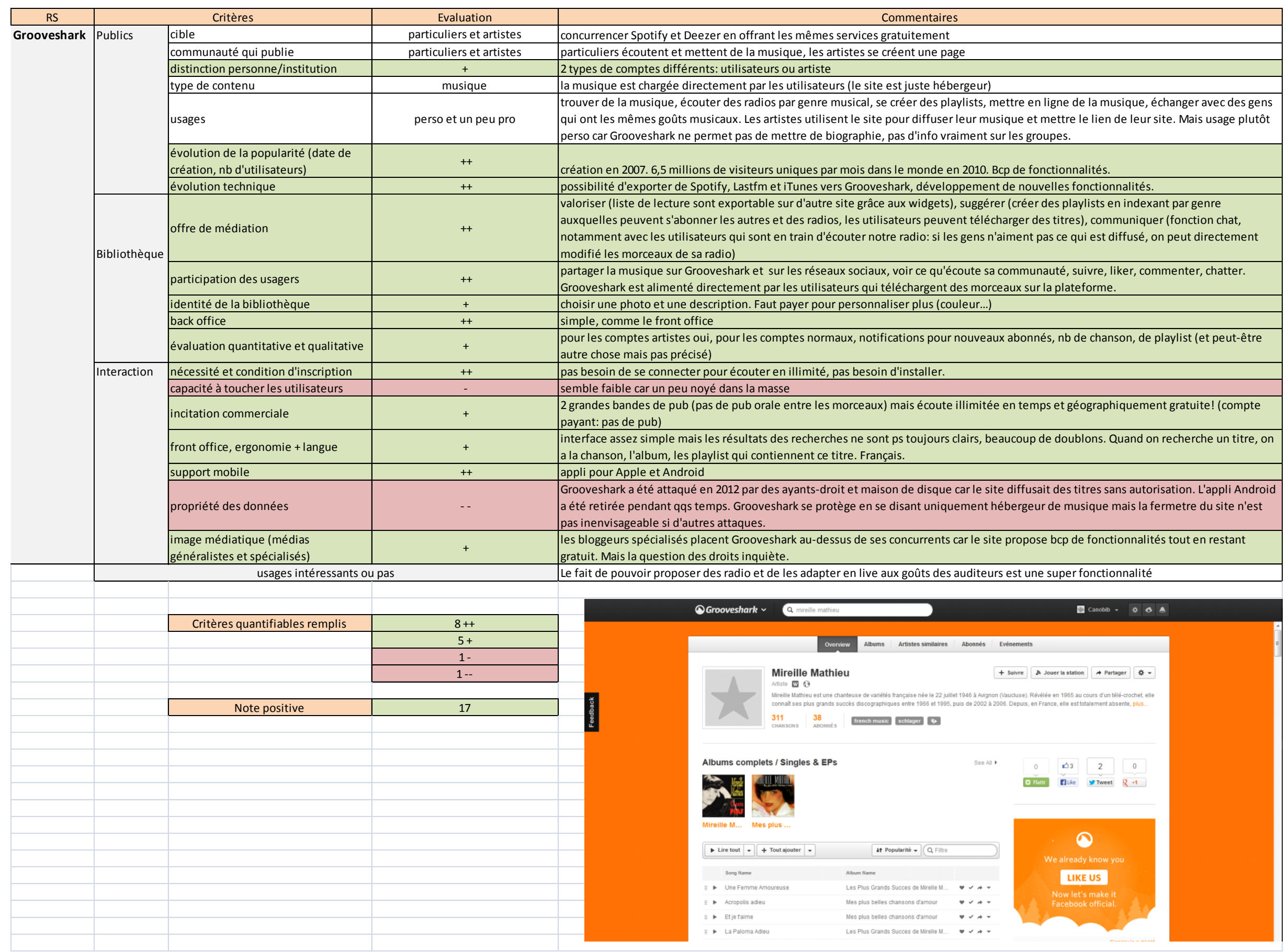




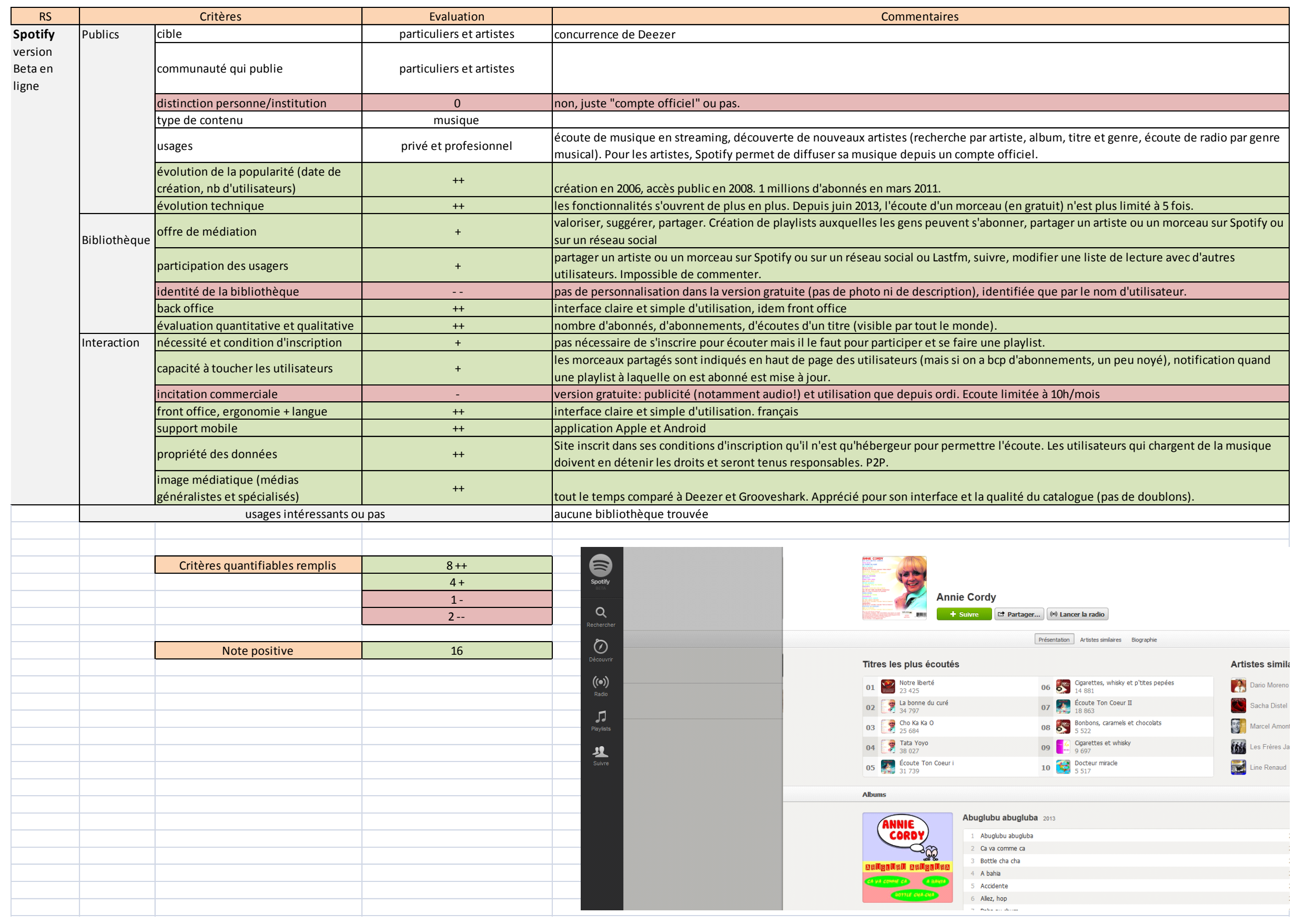




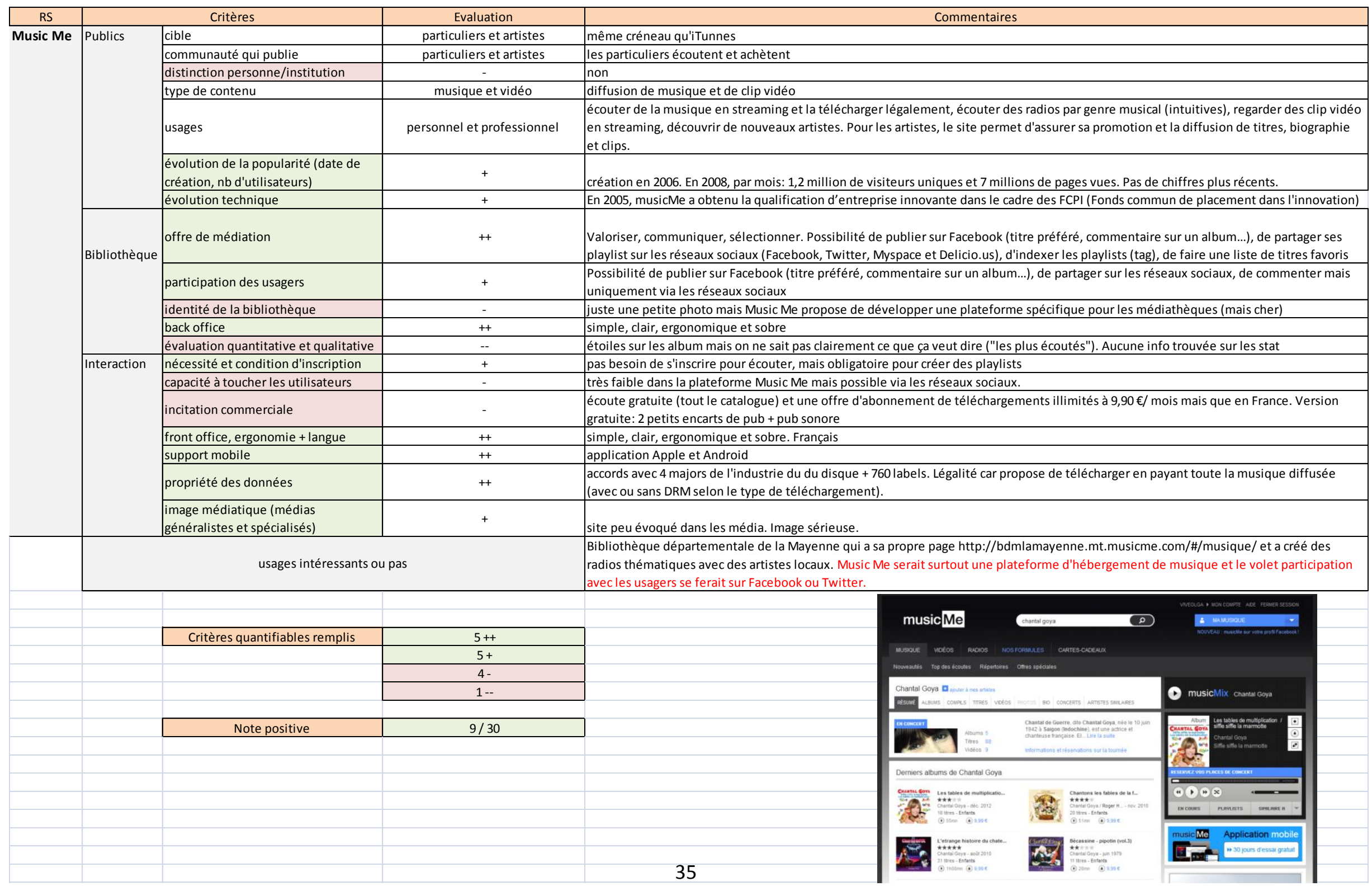




\begin{tabular}{|c|c|c|c|c|}
\hline RS & & Critères & Evaluation & Commentaires \\
\hline \multirow{19}{*}{ Pinterest } & \multirow{7}{*}{ Publics } & cible & entreprises et particuliers & $\begin{array}{l}\text { permettre aux marques de publier du visuel attractif et aux particuliers de partager leurs centres d'intérêts (souvent pour rediriger vers } \\
\text { leur blog). Pinterest est une sorte de vitrine. }\end{array}$ \\
\hline & & communauté qui publie & marques, femmes & les hommes sont de plus en plus nombreux tout de même \\
\hline & & distinction personne/institution & - & non, même page pour les particuliers et les institutions (seules indications: le nom de la page et la description) \\
\hline & & type de contenu & photo & réseau social + partage de photos (pin , épingler et interest, intérêt) \\
\hline & & usages & $\begin{array}{l}\text { féminin, commercial (marques), } \\
\text { les jeunes }\end{array}$ & $\begin{array}{l}\text { les utilisateurs aux USA sont très majoritairement des femmes ( } 70 \% \text { des utilisateurs il y a un an), pour mode, déco... mais tendance à une } \\
\text { réduction de l'écart hommes/femmes !!au Royaume-Uni: } 56 \% \text { sont des hommes et donc sujets différents!! } 30 \% \text { des utilisateurs ont entre } \\
25 \text { et } 34 \text { ans. Outil de curation perso. Pour les marques: Pinterest génère du trafic vers site marchand ou d'information. }\end{array}$ \\
\hline & & $\begin{array}{l}\text { évolution de la popularité (date de } \\
\text { création, nb d'utilisateurs) }\end{array}$ & $\begin{array}{l}\text { forte croissance récente, encore } \\
\text { peu répandu en France + }\end{array}$ & $\begin{array}{l}\text { création en 2010. 48,7 millions de membres dans le monde dont } 1 \text { million de visiteur unique en France. Augmentation ininterrompue des } \\
\text { utilisateurs }\end{array}$ \\
\hline & & évolution technique & ++ & pas de grandes avancées, ce qui permet à l'utilisateur de ne pas être surpris. Surtout des améliorations ergonomiques. \\
\hline & \multirow[t]{5}{*}{ Bibliothèque } & offre de médiation & + & $\begin{array}{l}\text { valoriser par l'image (un événement, une nouveauté, une thématique), décrire très succinctement par une légende (un document, une } \\
\text { expo, une animation), suggérer (en réépinglant d'autres pages) }\end{array}$ \\
\hline & & participation des usagers & & $\begin{array}{l}\text { possibilité de publier des photos sur son tableau perso, de liker/commenter/réépingler les photos des autres. Les utilisateurs ne peuvent } \\
\text { rien épingler sur le tableau de la bib mais ils peuvent liker sur FB et twitter. }\end{array}$ \\
\hline & & identité de la bibliothèque & - & $\begin{array}{l}\text { interface pratiquement pas personnalisable, juste une photo de profil et une rapide description (qqs lignes), lien vers site (institutionnel, } \\
\text { blog), Twitter et FB (+) }\end{array}$ \\
\hline & & back office & ++ & interface intuitive, peu de fonctionnalités, prise en main rapide, idem front office \\
\hline & & évaluation quantitative et qualitative & + & des stats sont proposées depuis mars 2013 uniquement pour les pages certifiées (compte relié à un site internet ou un blog vérifié) \\
\hline & \multirow[t]{7}{*}{ Interaction } & nécessité et condition d'inscription & - & $\begin{array}{l}\text { possibilité d'accéder aux pages sans inscriptions mais il faut connaître le nom de la page pour rentrer sur Pinterest (recherche Google). } \\
\text { obligation d'être inscrit pour interagir. A l'inscription, il faut sélectionner } 5 \text { personnes à suivre. }\end{array}$ \\
\hline & & capacité à toucher les utilisateurs & & $\begin{array}{l}\text { les photos publiées peuvent être noyées dans la masse: le tableau-timeline de l'utilisateur présente toutes les dernières photos publiées } \\
\text { par les autres en vrac, pas d'identification instantannée de l'émetteur. }\end{array}$ \\
\hline & & incitation commerciale & ++ & non, aucune publicité \\
\hline & & front office, ergonomie + langue & ++ & interface intuitive, peu de fonctionnalités, prise en main rapide. LANGUE: Français \\
\hline & & support mobile & ++ & appli android et apple \\
\hline & & propriété des données & - & $\begin{array}{l}\text { Tous les droits des œuvres originales sont cédés à Pinterest MAIS les images sont juste déposées sur Pinterest, elles restent sourcées avec } \\
\text { un lien qui redirige vers le site d'origine MAIS cela ne garantit par pour autant la légalité des droits. Seuls paramètres de confidentialité: } \\
\text { tableau public ou privé }\end{array}$ \\
\hline & & $\begin{array}{l}\text { image médiatique (médias } \\
\text { généralistes et spécialisés) }\end{array}$ & + & $\begin{array}{l}\text { la montée de la popularité du site intéressent les médias. Image positive: nouveauté, génère du trafic / image négative: juste un buzz? } \\
\text { Propriété des données cédées à Pinterest }\end{array}$ \\
\hline & \multicolumn{3}{|c|}{ usages intéressants ou pas } & $\begin{array}{l}\text { British Library et New York Public Library alimentent des albums par centres d'intérêts: les chats, la cuisine, la bibliothèque... Pas } \\
\text { uniquement centré sur l'offre documentaire = bonne idée à suivre. Bib Louise Michel: albums avec lère de couv des docs présentés au } \\
\text { "café de Louise" (café littéraire hebdomadaire à la bib) avec un lien vers la notice du catalogue. }\end{array}$ \\
\hline
\end{tabular}

\begin{tabular}{|c|c|}
\hline Critères quantifiables remplis & $5++/ 15$ \\
\hline \multirow{5}{*}{} & $5+/ 15$ \\
\cline { 2 - 2 } & $4-/ 15$ \\
\cline { 2 - 2 } & $2--/ 15$ \\
\hline
\end{tabular}

Note positive

8

Pinterest Ajouter + À prope

Pins · Tableaux · Utilisateurs - résultats de recherche

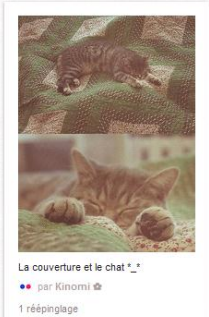

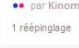

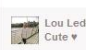

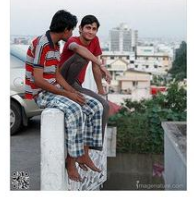

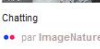

Cr. Phylis wartin arley

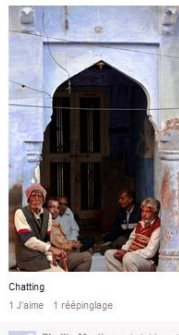

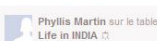

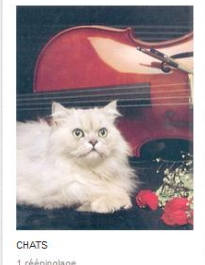

2. Giagsa Amarat sur te taveleay

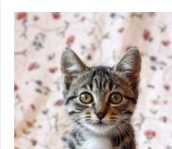

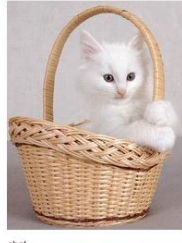

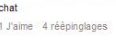

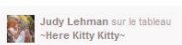

9:

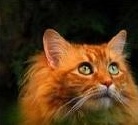

20.9
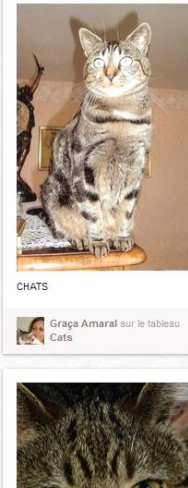


\begin{tabular}{|c|c|c|c|c|}
\hline RS & & Critères & Evaluation & Commentaires \\
\hline \multirow[t]{19}{*}{ Tumblr } & \multirow[t]{7}{*}{ Publics } & cible & pros élitistes & artistes, photographes, de créatifs et assimilés; marques élististes \\
\hline & & communauté qui publie & $\begin{array}{l}\text { ados, artistes, } \\
\text { marques }\end{array}$ & forte montée en puissance des jeunes et ados; média décalé avec .gif parodiques \\
\hline & & distinction personne/institution & - & non mais paramétrage de l'arrière plan possible \\
\hline & & type de contenu & $\begin{array}{l}\text { texte, photos, } \\
\text { vidéo, citations }\end{array}$ & texte, photos, vidéo, citations \\
\hline & & usages & blogging rapide & curation rapide; créations, liens, détournement, publications; \\
\hline & & $\begin{array}{l}\text { évolution de la popularité (date de } \\
\text { création, nb d'utilisateurs) }\end{array}$ & ++ & progression exponentielle; créé en 2007; 33 M de compte en 2011, 107 M en 2013; \\
\hline & & évolution technique & + & suivie \\
\hline & \multirow[t]{5}{*}{ Bibliothèque } & offre de médiation & + & couverture des documents; détournement de .gif; photos d'animation, partage de contenu \\
\hline & & participation des usagers & ++ & $\begin{array}{l}\text { possibilité d'aimer, de contribuer, partager, poser une question, avoir plusieurs auteurs sur des blogs } \\
\text { supplémentaires }\end{array}$ \\
\hline & & identité de la bibliothèque & + & personnalisation de l'arrière plan et "about me"; possibilité de paramétrer en html si on veut ! \\
\hline & & back office & ++ & très simple d'utilisation \\
\hline & & évaluation quantitative et qualitative & + & statistiques par google analytics \\
\hline & \multirow[t]{7}{*}{ Interaction } & nécessité et condition d'inscription & - & oui pour commenter etc; mais visible par tous \\
\hline & & capacité à toucher les utilisateurs & ++ & pas de contrainte de paiement pour toucher plus d'utilisateur; flux équivalent à twitter \\
\hline & & incitation commerciale & ++ & on paie seulement si on veut un arrière-plan plus sophistiqué \\
\hline & & front office, ergonomie + langue & + & $\begin{array}{c}\text { français; front office paramétrable pour autoriser commentaires, partages, questions etc; fonction } \\
\text { de recherche pas très au point par contre }\end{array}$ \\
\hline & & support mobile & ++ & oui \\
\hline & & propriété des données & -- & pas trouvé \\
\hline & & image médiatique & ++ & très favorable; popularité croissante, utilisé par créatifs; à la fois popu et elitiste \\
\hline & \multicolumn{3}{|c|}{ usages intéressants ou pas } & médiations vers documents et info décalée \\
\hline & & Critères quantifiables remplis & $7++$ & \multirow{6}{*}{$\begin{array}{l}\text { 11 La lionne blanche - } \\
\text { Henning Mankell }\end{array}$} \\
\hline & & & $5+$ & \\
\hline & & & $2-$ & \\
\hline & & & 1 -- & \\
\hline & & Note globale & 15 & \\
\hline & & & & \\
\hline
\end{tabular}




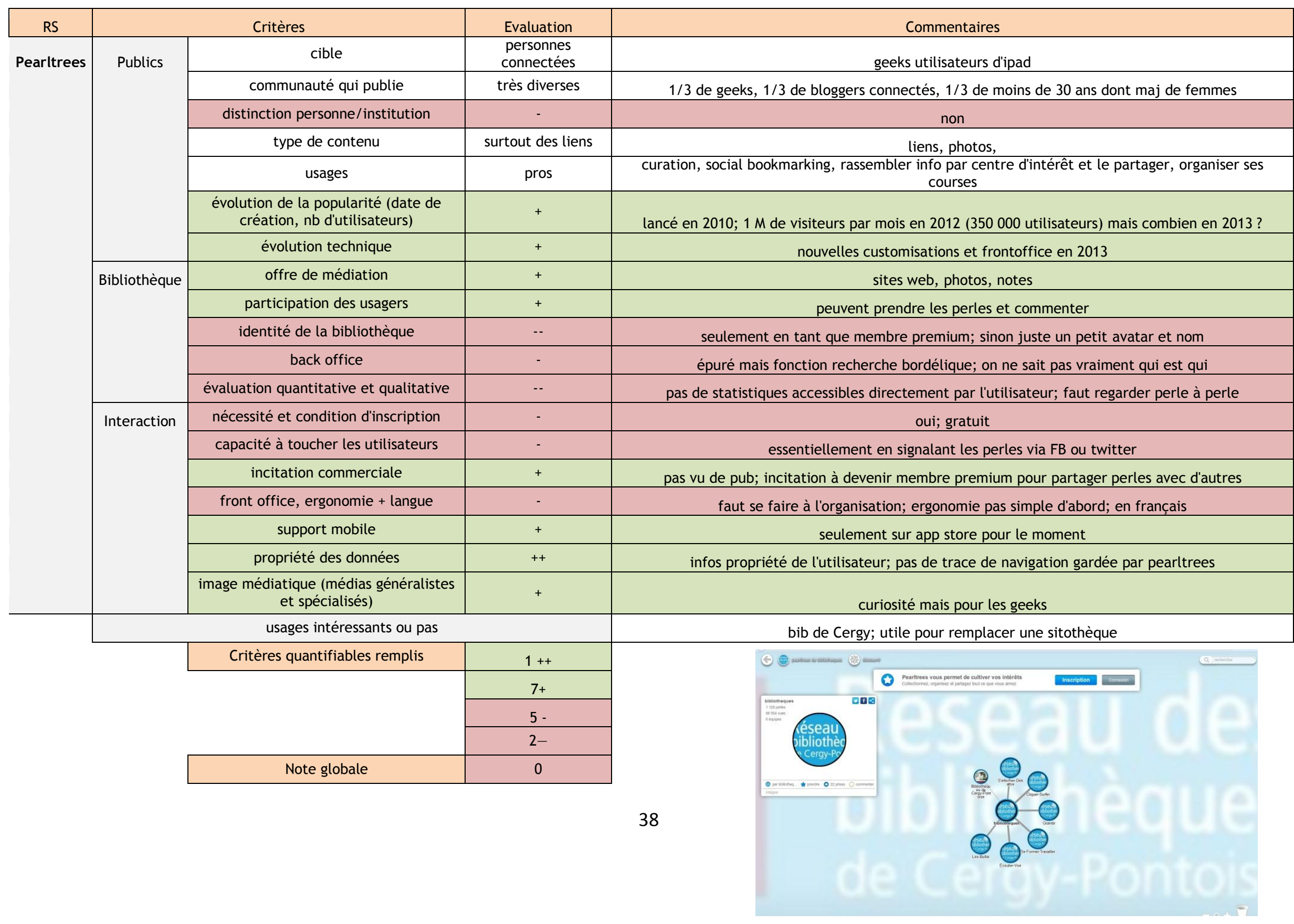




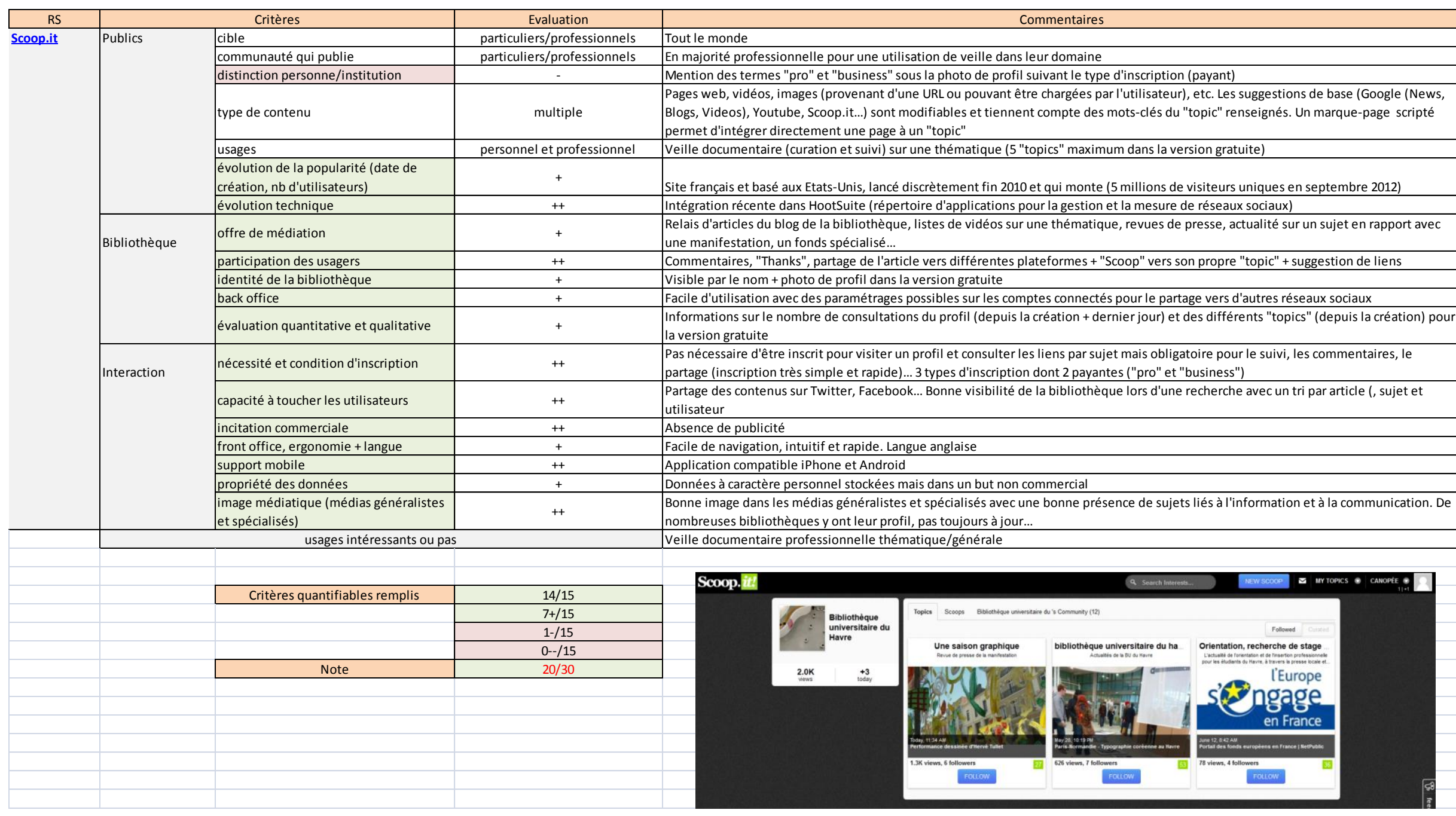




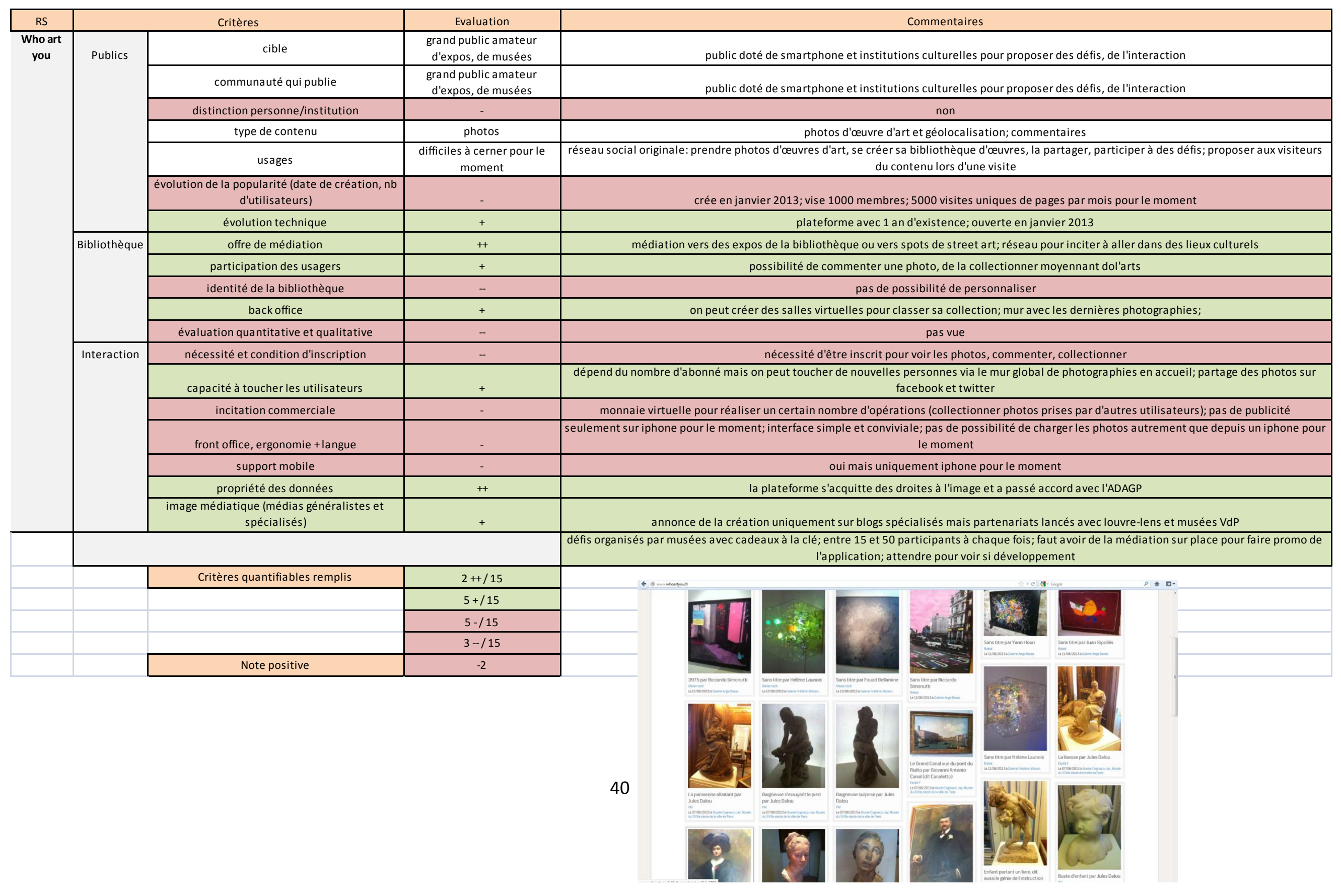




\begin{tabular}{|c|c|c|c|c|}
\hline \multirow{20}{*}{\begin{tabular}{|c|} 
RS \\
Babelio
\end{tabular}} & \multirow{8}{*}{ Publics } & \multirow[t]{2}{*}{ Critères } & Evaluation & Commentaires \\
\hline & & & Particuliers et jeunes & tout public internaute et assez gros ou très gros lecteurs ( $\mathrm{min}+\mathrm{d}$ '1 livre/mois), élèves des collèges et lycées \\
\hline & & communauté qui publie & gros lecteurs & hommes, femmes, des adultes principalement, quelques ados (rares), profs, quelques professionnels (minorité) \\
\hline & & distinction personne/institution & - & non, il est meme difficile de repérer l'institution \\
\hline & & type de contenu & texte & $\begin{array}{l}\text { réseau social et critique de livres des internautes et de presse et moteur de recherche de lecture par rapport a ce } \\
\text { qu'on aime }\end{array}$ \\
\hline & & usages & loisirs culturels & $\begin{array}{l}\text { recherche de nouvelles lectures, partage de ce qu'on a aimé, jeux et quiz, information en littérature, possibilité } \\
\text { d'ajouter des photos, vidéos et podcasts sur les auteurs uniquement }\end{array}$ \\
\hline & & $\begin{array}{l}\text { évolution de la popularité (date de } \\
\text { création, nb d'utilisateurs) }\end{array}$ & ++ & $\begin{array}{l}\text { lancé en 2007, } 58000 \text { lecteurs membres contributeurs en } 2012 \text { et } 830000 \text { à 1,3 million de visites en lecture seule. } \\
\text { Constante augmentation }\end{array}$ \\
\hline & & évolution technique & + & $\begin{array}{l}\text { adaptations constantes et nouvelles fonctionnalités en } 2013 \text { ex : création d'un catégorie babeldoc pour les profs } \\
\text { documentalistes, possibilité de lier son compte FB à babelio pour que les critiques soient lisibles sur FB, a tout } \\
\text { moment possibilité de référencer une page sur FB, Twitter ou google+ }\end{array}$ \\
\hline & Bibliothèque & offre de médiation & + & $\begin{array}{l}\text { mettre en ligne ses analyses, toucher un autre public physiquement plus éloigné de la bibliothèque, presenter des } \\
\text { coups de cœeurs, les regrouper sous une étiquette qui correspond à un temps fort. Pas de lien vers le catalogue dans } \\
\text { les notices. Aussi possibilité de repérer les membres référencés à paris } 75001 \text {, par exemple. }\end{array}$ \\
\hline & & participation des usagers & + & possibilité de commenter une critique ou un article, liker, pas de référencement d'autres pages dans son profil \\
\hline & & identité de la bibliothèque & -- & $\begin{array}{l}\text { interface assez figée a part une photo de profil, une description rapide un lien vers un blog ou site (reco } \\
\text { tweeter }\end{array}$ \\
\hline & & back office & -- & module babelthèque concu pour enrichir le sigb, les membres sont classés selon si pro ou particuliers \\
\hline & & évaluation quantitative et qualitative & - & pas d'évaluation systématique \\
\hline & Interaction & nécessité et condition d'inscription & + & $\begin{array}{l}\text { inscription pour publier et obtenir des suggestions de lectures personnalisées mais possibilité de consulter des } \\
\text { publications sans inscription. }\end{array}$ \\
\hline & & capacité à toucher les utilisateurs & - & Comparatifs : mieux que goodread et livraddict, le nombre de livres en commun augmente la visibilité \\
\hline & & incitation commerciale & + & $\begin{array}{l}\text { lien avec Amazon pour acheter les livres qui plaisent, financement par des pub d'éditeur et service babelthèque } \\
\text { vendu aux bibliothèques }\end{array}$ \\
\hline & & front office, ergonomie & ++ & très simple d'utilisation et accrocheur. convient à tous. \\
\hline & & support mobile & -- & non \\
\hline & & propriété des données & + & le contenu mis sur BaBelio est propriété de Babelio tant que son auteur le laisse sur le site (hebergement) \\
\hline & & $\begin{array}{l}\text { image médiatique (médias } \\
\text { généralistes et spécialisés) }\end{array}$ & + & $\begin{array}{l}\text { propose les critiques de la presse littéraire généraliste et spécialisée, présent avec un blog sur word press, sur FB, sur } \\
\text { twitter, sur pinterest, sur pearltrees }\end{array}$ \\
\hline & \multicolumn{3}{|c|}{ usages intéressants ou pas } & $\begin{array}{l}\text { Contenu proposé par les médiathèques assez pauvre (voir MMY = Yourcenar) pas de présence lié à l'actualité sur le site } \\
\text { et pas de visibilité. II semblerait que les médiathèques mettent peu à jour ce média. Les internautes aiment, la presse } \\
\text { le référence peu mais babelio reprend toute la presse littéraire aussi les éditeurs l'utilisent beaucoup. }\end{array}$ \\
\hline & & Critères quantifiables remplis & $2++$ & 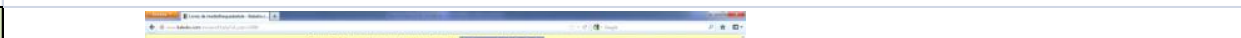 \\
\hline & & & $7+$ & 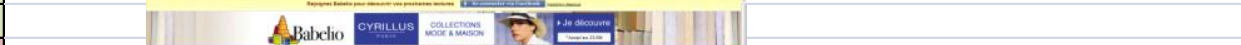 \\
\hline & & & $3-$ & 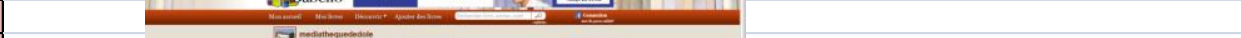 \\
\hline & & & 3-- & $=-1-\ldots$ \\
\hline & & & & 20 \\
\hline & & Note positive & 2 & 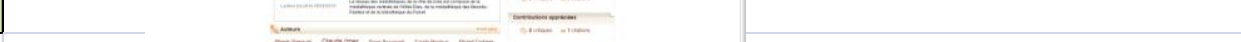 \\
\hline & & & & $\equiv=$ \\
\hline & & & & $=-=-1$ 圆 \\
\hline & & & & $=0$ \\
\hline
\end{tabular}




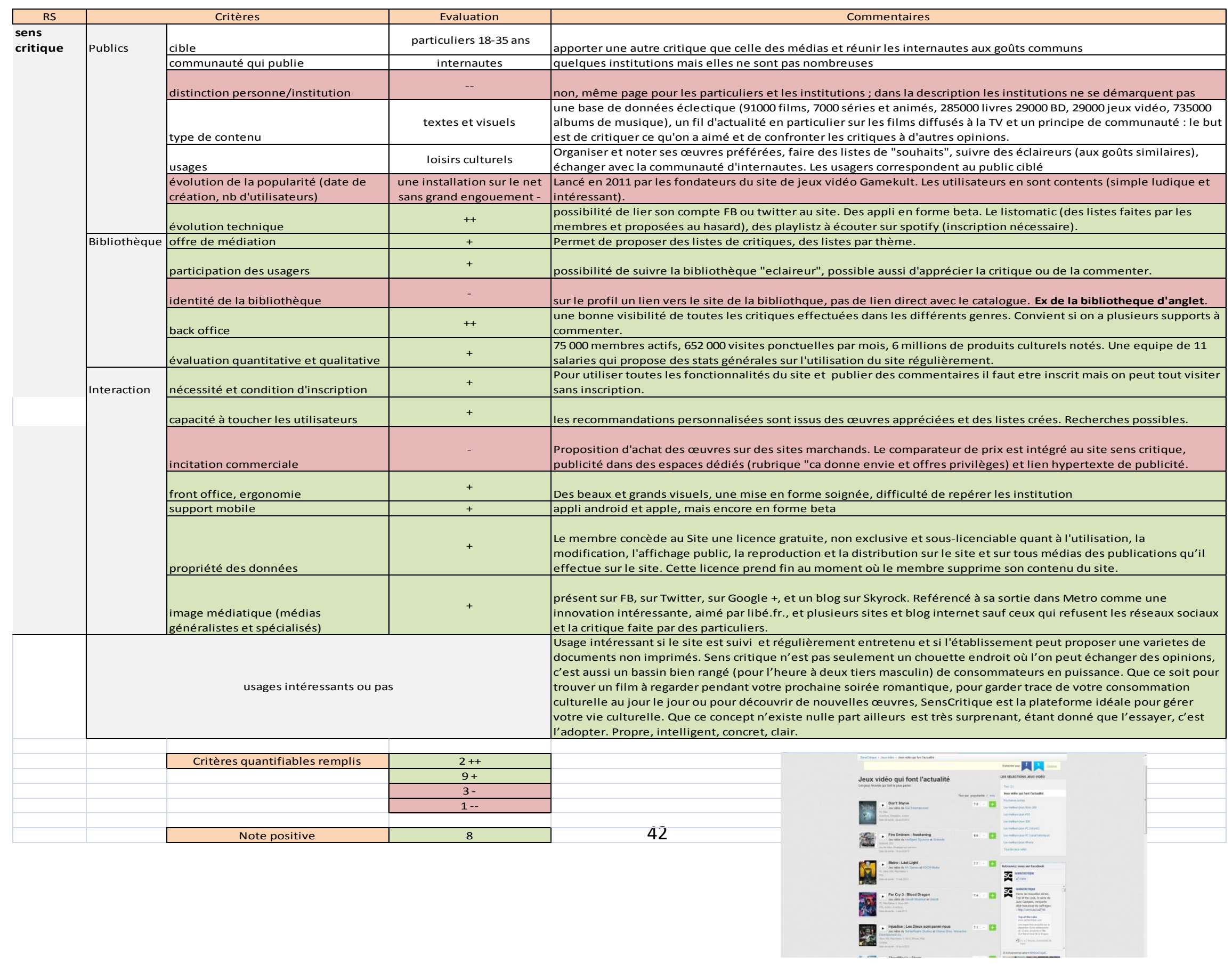




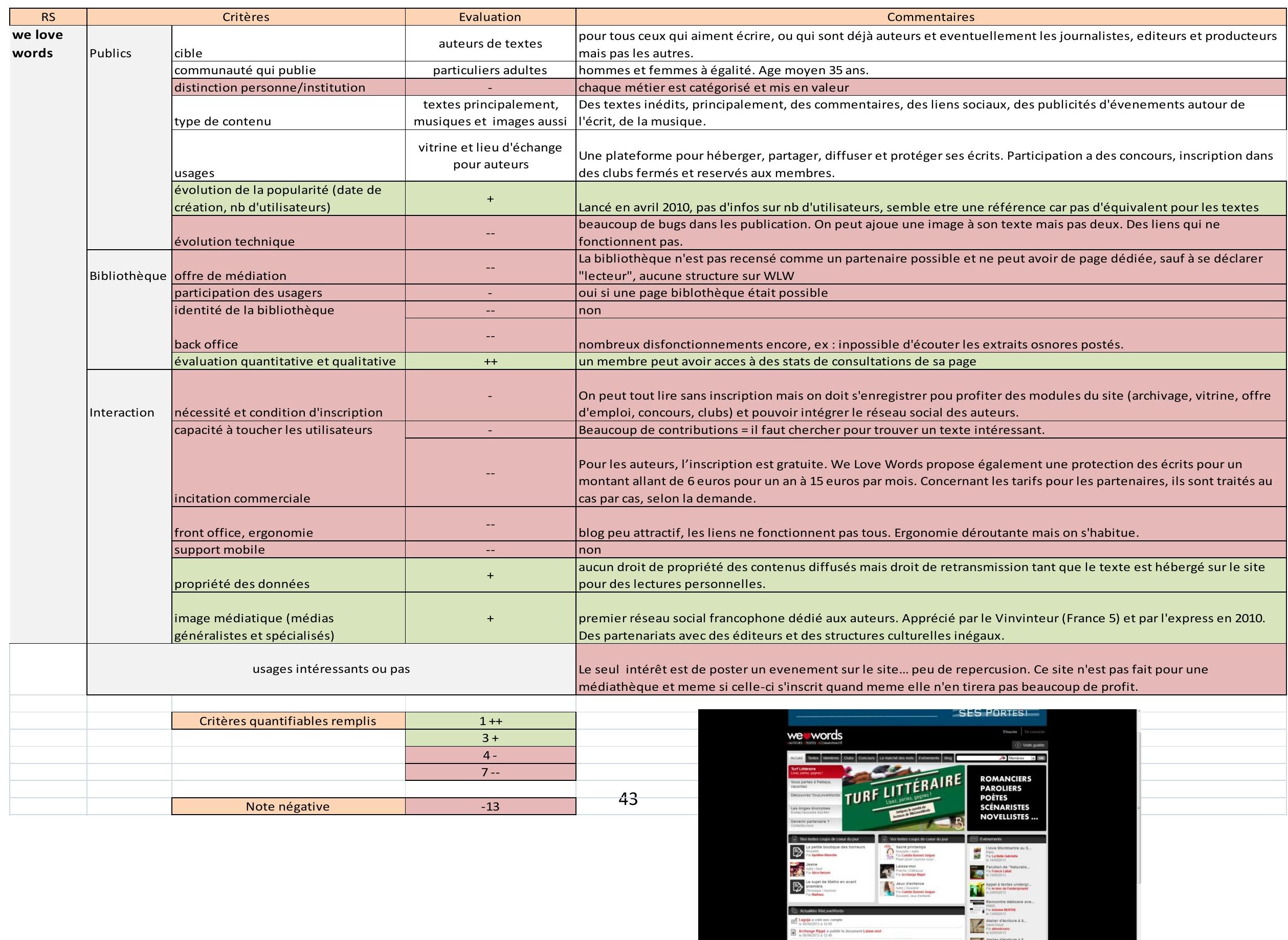




\begin{tabular}{|c|c|c|c|c|}
\hline RS & & Critères & Evaluation & Commentaires \\
\hline \multirow[t]{19}{*}{ Riffle } & \multirow[t]{7}{*}{ Publics } & cible & particuliers anglophones & Particuliers qui souhaitent partager leurs lectures \\
\hline & & communauté qui publie & particuliers adultes & les membres sont caractérisés 1 par le genre qu'il aime/ 2 par leur lieu d'habitation (ville + etat pour les US) \\
\hline & & distinction personne/institution & -- & institutions absentes et/ou introuvables, les personnes sont mentionnées par leurs noms et prénoms \\
\hline & & type de contenu & $\begin{array}{l}\text { listes de livres pas de } \\
\text { textes, pas de } \\
\text { commentaires }\end{array}$ & \\
\hline & & usages & suggestion de lectures & \\
\hline & & $\begin{array}{l}\text { évolution de la popularité (date de } \\
\text { création, nb d'utilisateurs) }\end{array}$ & + & création en 2013 , utilisateurs américains chiffres non communiqué \\
\hline & & évolution technique & -- & $\begin{array}{l}\text { une base de données à completer, un système de listes inté"ressant à l'image de Pinterest sans plus selon les } \\
\text { utilisateurs }\end{array}$ \\
\hline & \multirow[t]{5}{*}{ Bibliothèque } & offre de médiation & -- & pas de structure culturelles recencées \\
\hline & & participation des usagers & -- & pas de participation pas de texte juste à suivre \\
\hline & & identité de la bibliothèque & -- & non \\
\hline & & back office & -- & \\
\hline & & évaluation quantitative et qualitative & -- & non \\
\hline & \multirow[t]{7}{*}{ Interaction } & nécessité et condition d'inscription & -- & $\begin{array}{l}\text { Il faut etre inscrit pour consulter le site, l'inscription se fait uniquement via FB ou Tweeter et riffle demande un acces } \\
\text { automatique aux amis et aux publications, j'aime etc. }\end{array}$ \\
\hline & & capacité à toucher les utilisateurs & -- & via les listes porposées mais rien de probant : on ne peut rien cibler \\
\hline & & incitation commerciale & ++ & aucune, c'est vraiment un site de particuliers à particuliers \\
\hline & & front office, ergonomie & - & un peu brouillon : des livres à épingler, des listes à proposer, comme pinterest mais peu de fonctionnalités au final \\
\hline & & support mobile & -- & non \\
\hline & & propriété des données & -- & pas de données. \\
\hline & & $\begin{array}{l}\text { image médiatique (médias } \\
\text { généralistes et spécialisés) }\end{array}$ & -- & aucune, c'est vraiment un site de particuliers à particuliers \\
\hline & \multicolumn{3}{|c|}{ usages intéressants ou pas } & $\begin{array}{l}\text { aucun interet pour une bibliothèque car aucune visibilité de la structure. Aucun moyen de poster des commentaires. } \\
\text { Juste un lien vers un blog ou site ou page FB, peu de livre à suggérer et américains pour la plupart. Vraiment peu de } \\
\text { livres francais. }\end{array}$ \\
\hline & & Critères quantifiables remplis & $1++$ & Riffle $-\ldots \ldots+\ldots$ \\
\hline & & & $1+$ & $w-m-m$ \\
\hline & & & $1-$ & Fin \\
\hline & & & $12--$ & \\
\hline & & Note négative & -23 & (n) \\
\hline \multicolumn{5}{|r|}{$-\frac{1}{000}$} \\
\hline
\end{tabular}




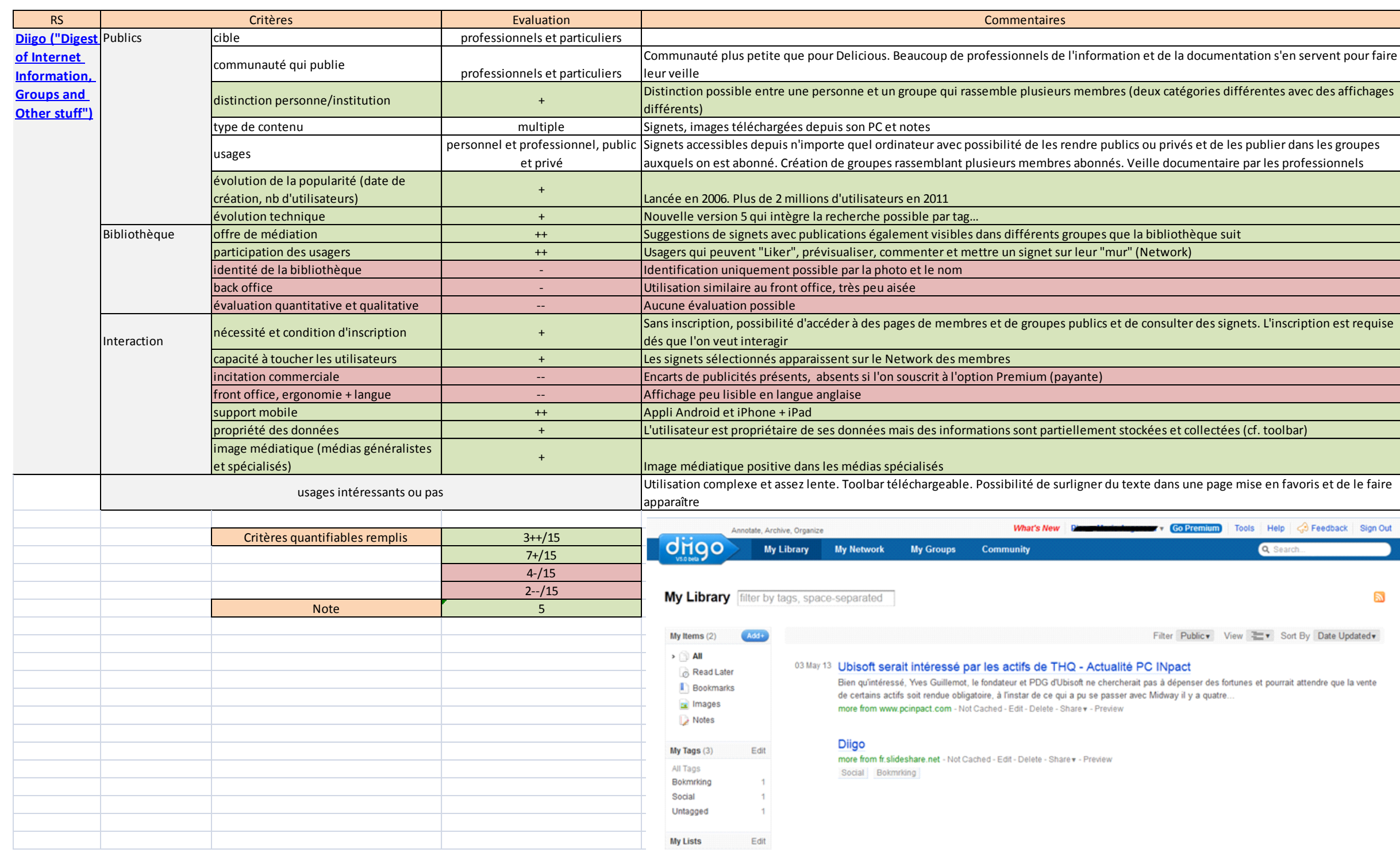




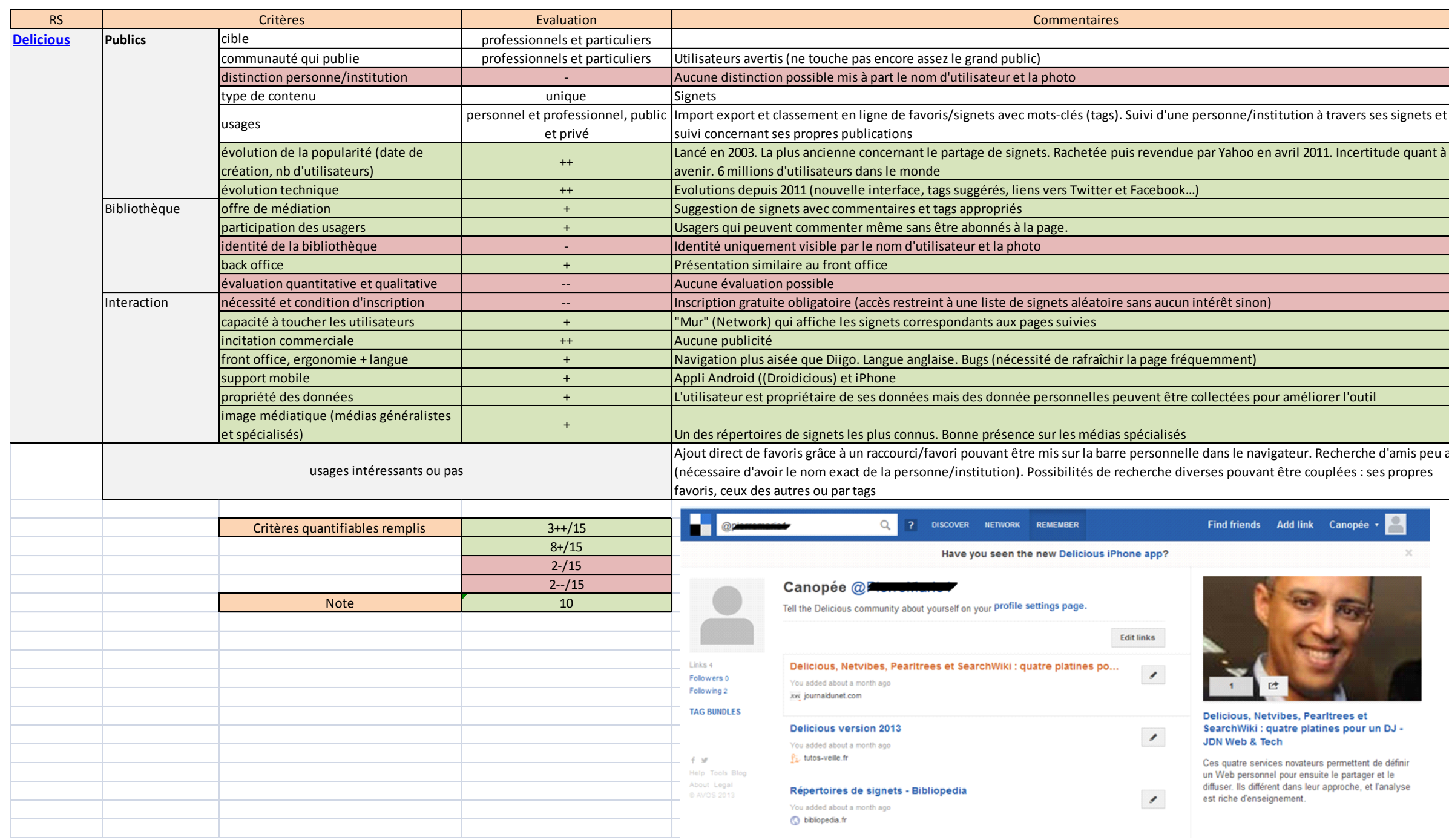




\begin{tabular}{|c|c|c|c|c|c|c|c|}
\hline \multirow{20}{*}{$\begin{array}{r}\text { RS } \\
\text { Netvibes } \\
\end{array}$} & \multirow{8}{*}{ Publics } & Critères & \multirow{2}{*}{$\begin{array}{c}\text { Evaluation } \\
\text { particuliers/professionnels }\end{array}$} & \multirow{2}{*}{\multicolumn{4}{|c|}{ Commentaires }} \\
\hline & & cible & & & & \multicolumn{2}{|c|}{ Tout le monde } \\
\hline & & communauté qui publie & particuliers/professionnels & \multicolumn{4}{|c|}{ Utilisé le plus par des professionnels qui organisent leur veille sur le web } \\
\hline & & distinction personne/institution & + & \multirow{2}{*}{\multicolumn{4}{|c|}{\begin{tabular}{|l|l} 
Possible à partir du nom du Netvibes et de la photo de profil \\
Liens Internet et contenus multibédias (vidéso )
\end{tabular}}} \\
\hline & & type de contenu & multiple & & & & \\
\hline & & usages & $\begin{array}{c}\text { personnel et professionnel, public } \\
\text { et privé }\end{array}$ & \multicolumn{4}{|c|}{$\begin{array}{l}\text { Liens Internet et contenus multimédias (vidéos...) } \\
\text { c Veille documentaire par l'agrégation de flux en ligne (ajout de flux RSS, widgets, applications...) organisés par applications, onglets et } \\
\text { dashboards (tableaux de bord le plus souvent thématiques) à l'intérieur d'un même compte }\end{array}$} \\
\hline & & $\begin{array}{l}\text { évolution de la popularité (date de } \\
\text { création, nb d'utilisateurs) }\end{array}$ & ++ & \multicolumn{4}{|c|}{$\begin{array}{l}\text { Lancé en } 2005 \text { (racheté par Dassault Systèmes en 2012) et rassemblant actuellement plus de } 4 \text { millions d'utilisateurs, essentiellement des } \\
\text { "particuliers" (cf. offre gratuite) qui peuvent être des professionnels }+1 / 2 \text { milliers de comptes premium }\end{array}$} \\
\hline & & évolution technique & + & \multicolumn{4}{|c|}{ Evolution continue suite au rachat par Dassault en 2012} \\
\hline & \multirow[t]{5}{*}{ Bibliothèque } & offre de médiation & + & \multicolumn{4}{|c|}{$\begin{array}{l}\text { Sélection de ressources Internet sur un sujet à la manière d'une sitothèque. Widgets permettant de communiquer mais de manière très } \\
\text { simpliste }\end{array}$} \\
\hline & & participation des usagers & - & \multicolumn{4}{|c|}{ Partage possible des liens vers leur page Facebook, Twitter ou Netvibes } \\
\hline & & identité de la bibliothèque & - & \multicolumn{4}{|c|}{ Par le nom et la photo (petite taille) avec la possibilité de jouer sur certains liens pour une meilleure visibilité (site de la ville...) } \\
\hline & & back office & + & \multicolumn{4}{|c|}{$\begin{array}{l}\text { Facile d'utilisation et de paramétrage } \\
\text { L'offre oratuite ne permet ancune analyse (voir conditions d'inscriptions) }\end{array}$} \\
\hline & & évaluation quantitative et qualitative & -- & \multicolumn{4}{|c|}{ L'offre gratuite ne permet aucune analyse (voir conditions d'inscriptions) } \\
\hline & \multirow[t]{7}{*}{ Interaction } & nécessité et condition d'inscription & + & \multicolumn{4}{|c|}{$\begin{array}{l}\text { Trois choix suivant que l'on est une agence, une entreprise ou une personne lambda (seul cas ou l'inscription est gratuite). Dans le dernier } \\
\text { cas, possibilité de payer pour avoir droit à une assistance à la création du dashboard, des analyses sociales personnalisées ou des alertes } \\
\text { en temps réel }\end{array}$} \\
\hline & & capacité à toucher les utilisateurs & -- & & & & \\
\hline & & incitation commerciale & ++ & \multicolumn{4}{|l|}{ Aucune même en version gratuite } \\
\hline & & front office, ergonomie + langue & + & \multirow{2}{*}{\multicolumn{4}{|c|}{$\begin{array}{l}\text { Facile et rapide d'utilisation. Langue française. Intuitif } \\
\text { Dispo sousiphone et Android }\end{array}$}} \\
\hline & & support mobile & + & & & & \\
\hline & & propriété des données & + & \multicolumn{4}{|c|}{ Données à caractère personnel stockées pendant un an mais aucune utilisation commerciale } \\
\hline & & $\begin{array}{l}\text { image médiatique (médias généralistes } \\
\text { et spécialisés) }\end{array}$ & ++ & \multicolumn{4}{|c|}{ Bonne image médiatique de la part des professionnels comme des particuliers (près de 10000 amis sur leur page Facebook) } \\
\hline & \multicolumn{3}{|c|}{ usages intéressants ou pas } & \multicolumn{4}{|l|}{ Forme de sitothèque } \\
\hline & & & & & & & . \\
\hline & & Critères quantifiables remplis & $3++/ 15$ & 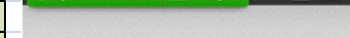 & 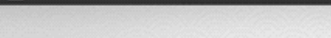 & 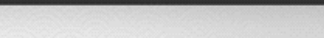 & \\
\hline & & & $8+/ 15$ & ( & 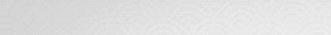 & ( & \\
\hline & & & $2-/ 15$ & (n) & 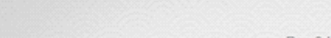 & 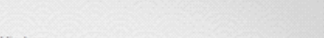 & \\
\hline & & & $2--/ 15$ & 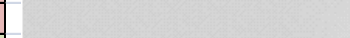 & Rap at & Hip hop & \\
\hline & & Note & 8 & & & $\sin \cos$ & \\
\hline & & & & 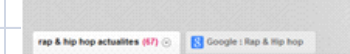 & & & \\
\hline & & & & 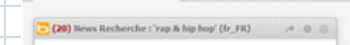 & 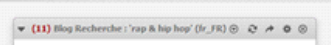 & 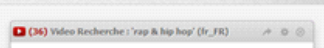 & somentemit \\
\hline & & & & 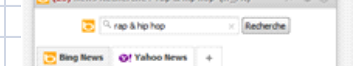 & 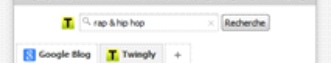 & 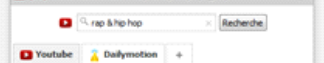 & 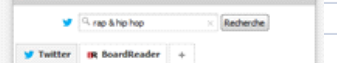 \\
\hline & & & & 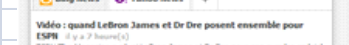 & 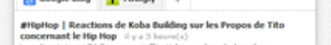 & 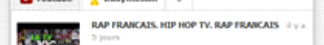 & 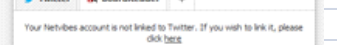 \\
\hline & & & & 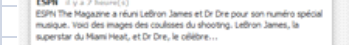 & 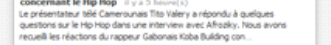 & & \\
\hline & & & & 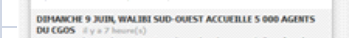 & 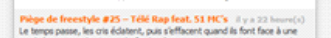 & & \\
\hline & & & & 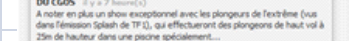 & 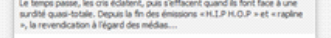 & & \\
\hline & & & & 然 & 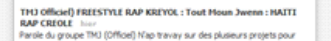 & & \\
\hline & & & & 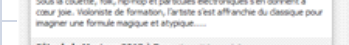 & 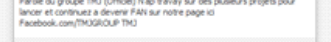 & & \\
\hline & & & & 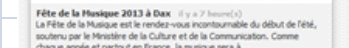 & 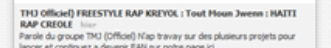 & Exis a & \\
\hline & & & & 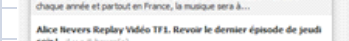 & 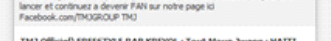 & $=2$ & \\
\hline & & & & 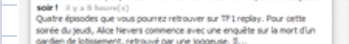 & 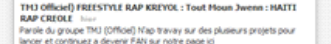 & (a) & \\
\hline & & & & $\ldots$ & & & \\
\hline
\end{tabular}




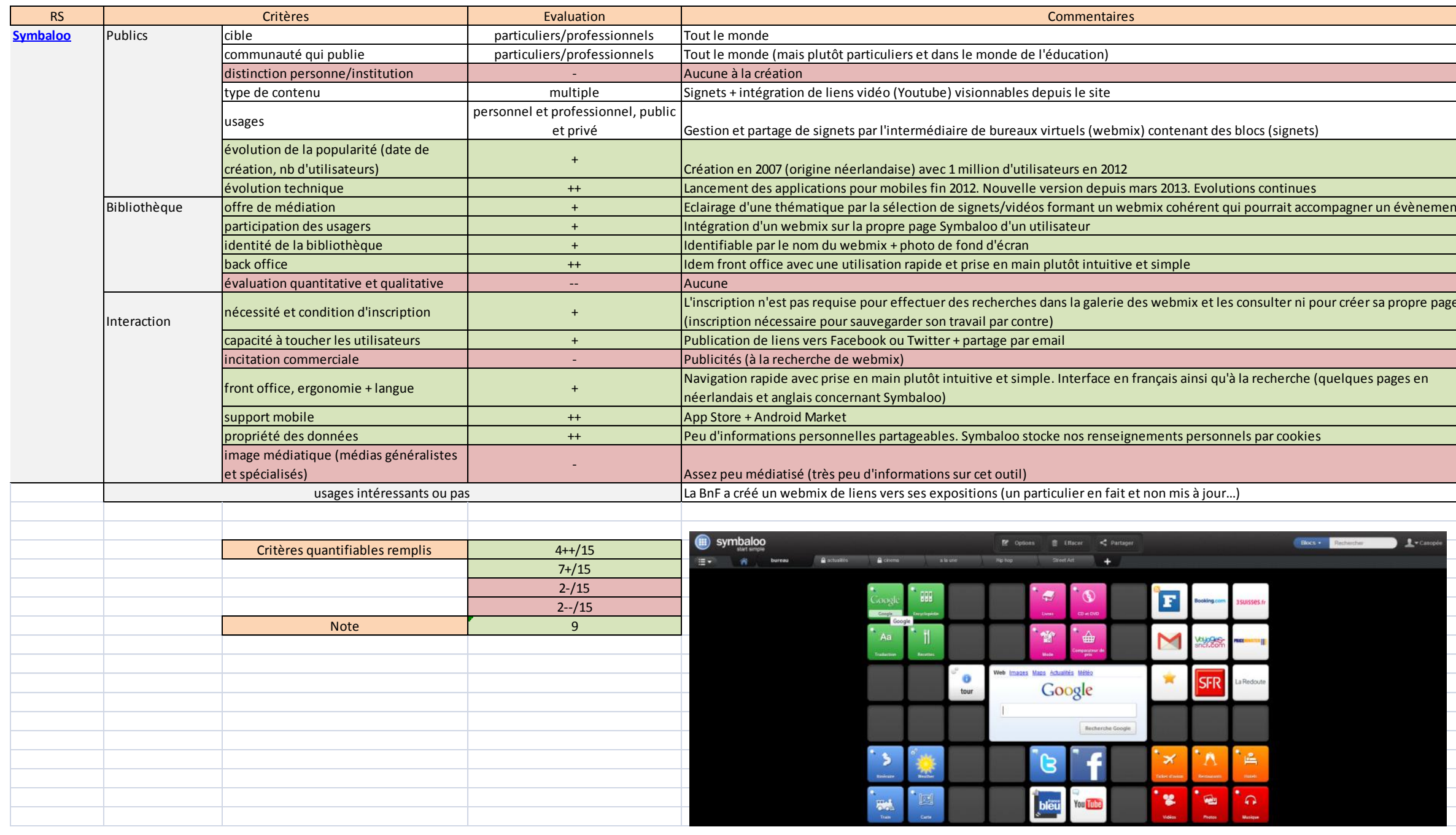




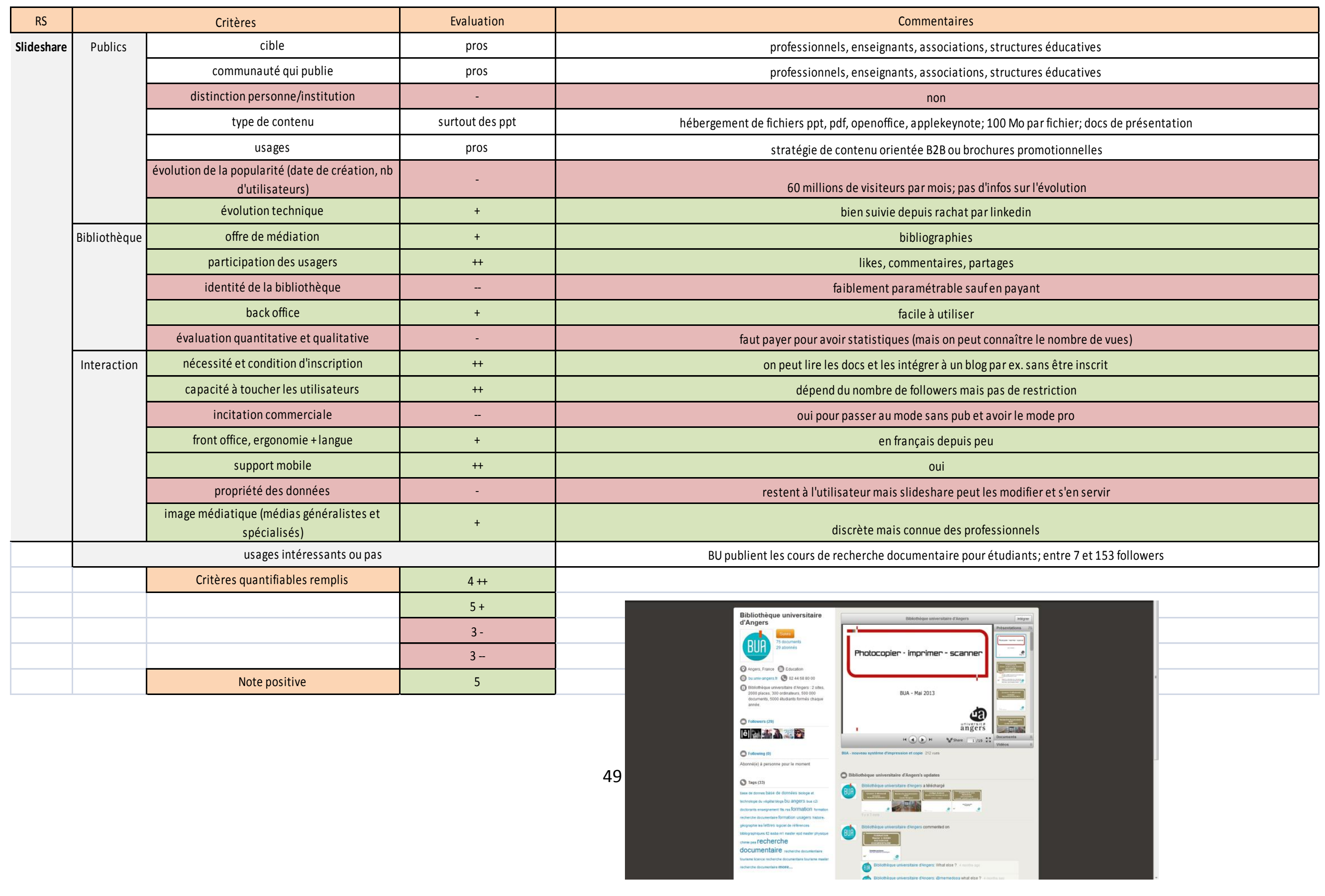




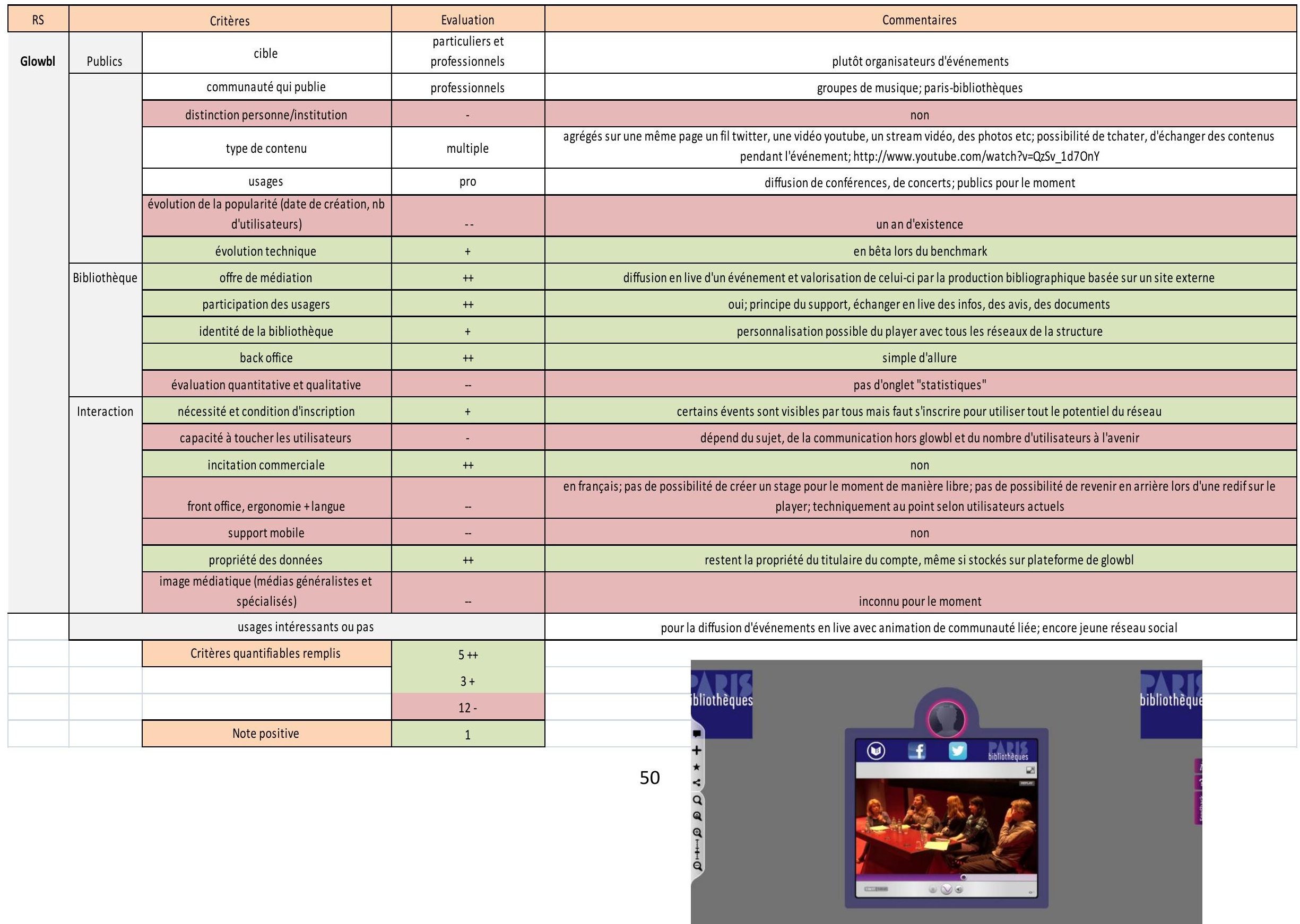




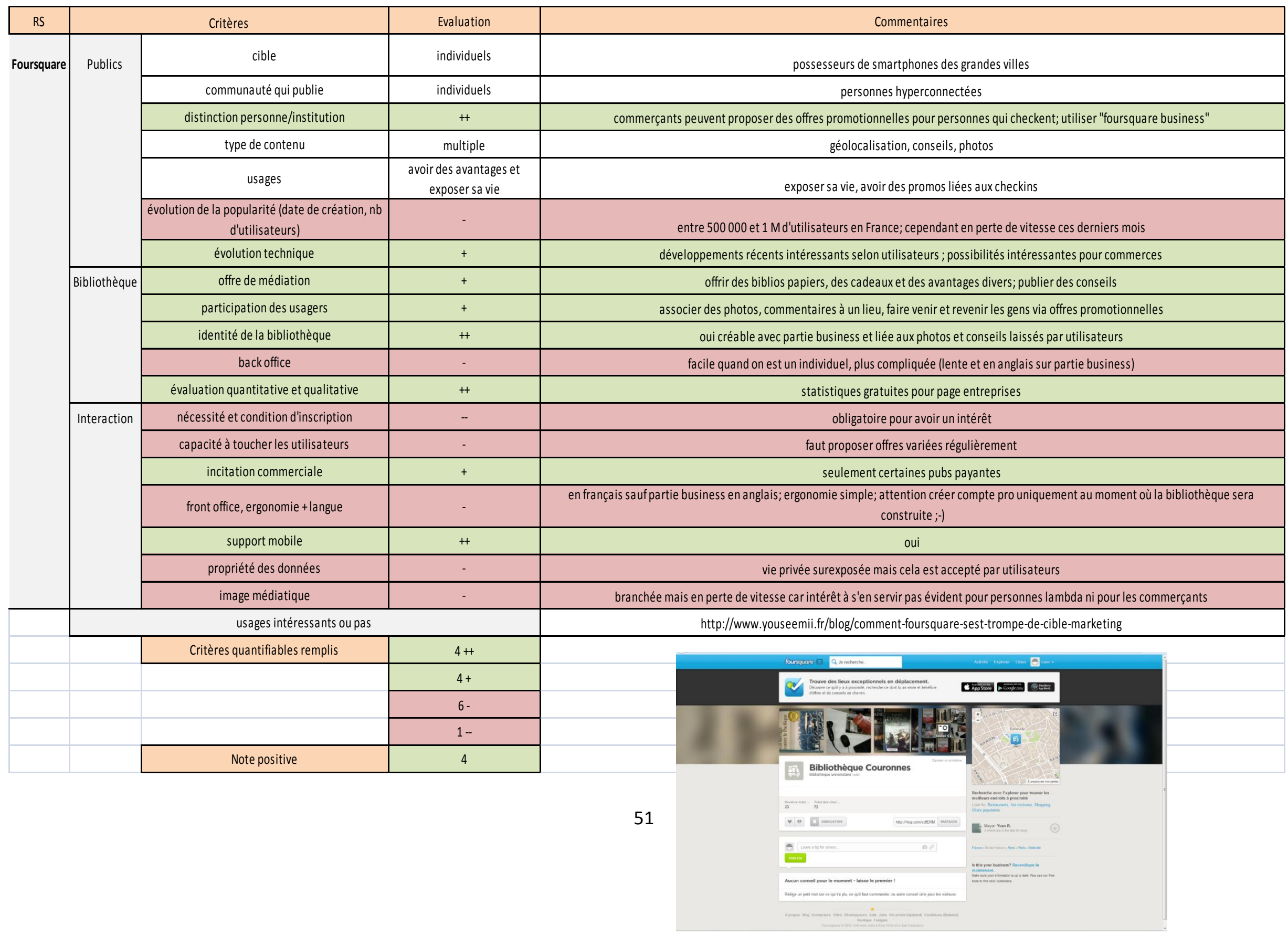




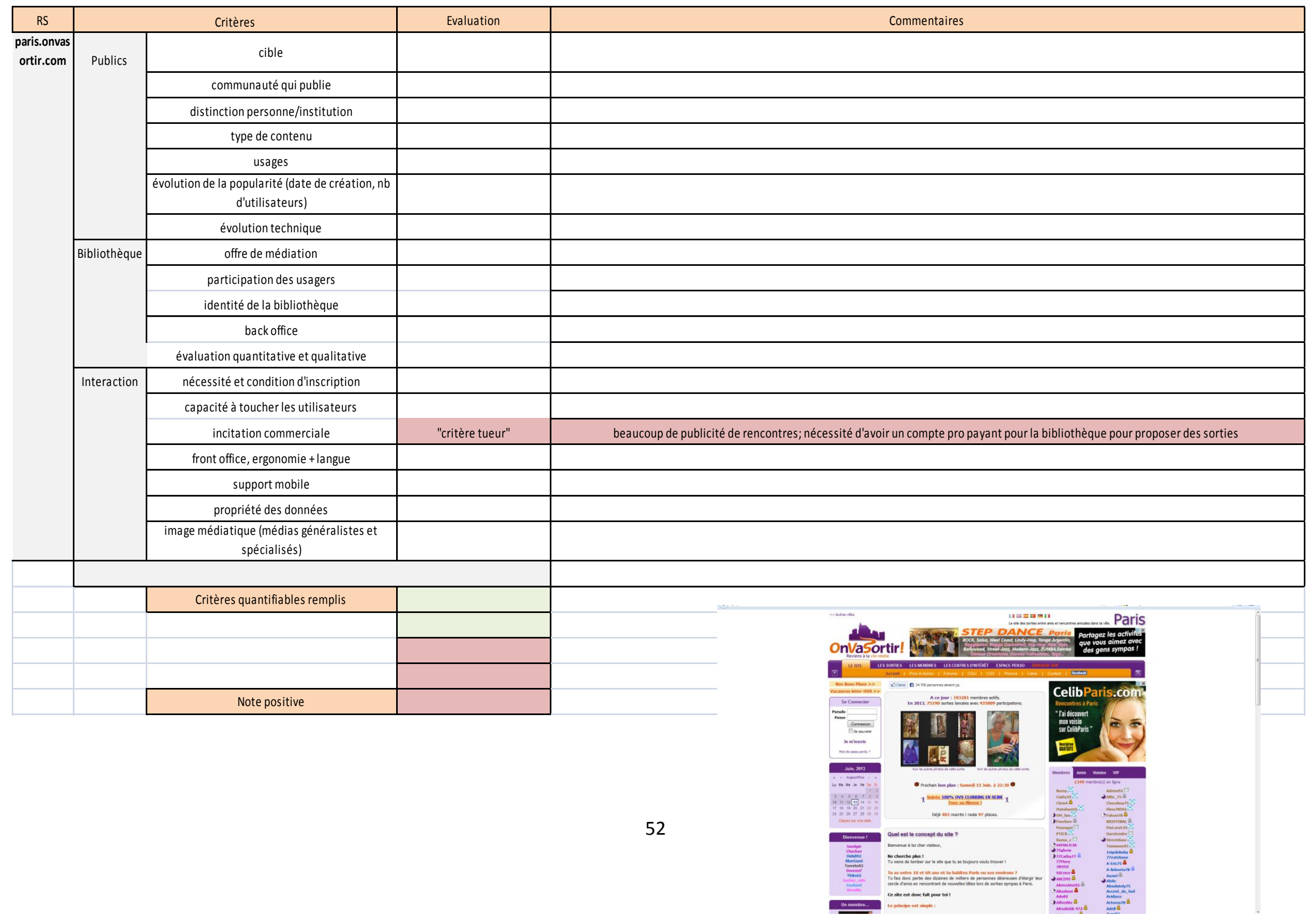


Etude coordonnée par la préfiguration de la bibliothèque Canopée et rédigée par: Pierre-Marie Augereau, Cyrille Engel, Romain Gaillard, Ophélie Hamot-Béchay, Capucine Liébeaux (préfiguration Canopée), Soizic Cadio (préfiguration SaintLazare) 